\title{
COMPUTATION OF MAASS WAVEFORMS WITH NONTRIVIAL MULTIPLIER SYSTEMS
}

\author{
FREDRIK STRÖMBERG
}

\begin{abstract}
The aim of this paper is to describe efficient algorithms for computing Maass waveforms on subgroups of the modular group $P S L(2, \mathbb{Z})$ with general multiplier systems and real weight. A selection of numerical results obtained with these algorithms is also presented. Certain operators acting on the spaces of interest are also discussed. The specific phenomena that were investigated include the Shimura correspondence for Maass waveforms and the behavior of the weight- $k$ Laplace spectra for the modular surface as the weight approaches 0 .
\end{abstract}

\section{INTRODUCTION AND NOTATION}

The purpose of the present paper is to present computational methods and experimental results for Maass waveforms with general real weight and general multiplier systems.

The classical theory of holomorphic automorphic forms was developed in the setting of (even) integer weight. This was motivated both from a geometrical point of view and by number theoretical applications (e.g. the study of modular forms related to the modular invariant $j$ and the discriminant $\Delta$ ). The problem of finding the number of representations of an integer as a sum of a fixed number of squares (cf. e.g. [45] and [19]) was successfully treated by using the theory of half-integral weight forms (e.g. $\theta$-series). This motivated Petersson to develop a theory of automorphic forms and multiplier systems of arbitrary real weight 52 and later also complex weight [54, I-IV].

Many applications of modular forms use Hecke operators as a principal tool, and Wohlfahrt developed a theory of Hecke-like operators in arbitrary real weight [76] (cf. also [70]).

Now recall the definition of a Maass waveform as a real-analytic square-integrable eigenfunction of the Laplacian on a Riemann surface of finite volume with constant negative curvature -1 . These were introduced by Maass for zero weight in 40. In [41] the theory of waveforms for general weights was developed by using the so-called lowering and raising operators, which send a waveform of a given weight to one with a smaller or larger weight.

In a slightly different setting Selberg [58, pp. 82-83] observed that considering the invariant differential operators on the space $\mathcal{H} \times S^{1}$ with representation $\chi$

Received by the editor November 3, 2006 and, in revised form, February 20, 2007.

2000 Mathematics Subject Classification. Primary 11-04; Secondary 11F72, 11F37.

Key words and phrases. Maass waveforms, multiplier systems, computational spectral theory, Shimura correspondence, Hecke operators.

(C)2008 American Mathematical Society Reverts to public domain 28 years from publication 
and separating variables lead to real analytic eigenfunctions of the form $f(z, \phi)=$ $y^{\frac{k}{2}} F(z) e^{-i k \phi}$, where $F(z)$ is a holomorphic modular form of integer weight $k$ and character $\chi$.

For an overview of the spectral theory of real analytic modular forms with arbitrary real weights and multiplier systems, see e.g. Maass [42] and Roelcke [56]. More recent work can be found in e.g. [49, 50, 51, [22, 23] and [8, 9, 10].

It is also worth mentioning that the recent interest in Maass waveforms as representative objects for studying quantum chaos also applies to real weights. If a weight zero waveform corresponds to a quantum mechanical particle moving freely on a Riemann surface, then a weight $k$ waveform represents a similar particle moving in a constant magnetic field with field strength proportional to $k$.

1.1. Algorithms. Previously published algorithms for computing Maass waveforms on cofinite Fuchsian groups have been restricted to groups with one cusp, e.g. the full modular group or Hecke triangle groups (see e.g. 63, 75, 25, 26, 27, 46]). By adjoining certain elements it is also possible to bring certain Hecke congruence subgroups to the one-cusp case, cf. e.g. [16, 17, 4].

In addition, with the exception of the (somewhat crude) computations in [46], only the trivial multiplier system and zero (or even integer for the holomorphic case in [26]) weight have been considered.

The most stable of the algorithms cited above is the one based on "implicit automorphy" by Hejhal (as detailed in e.g. [27]) which admits generalizations first of all to a remarkably large spectral parameter (cf. 69]), and a further advantage is that it does not depend on any underlying arithmetical properties (i.e. Hecke operators).

In 65 this algorithm was generalized to groups with several cusps e.g. Hecke congruence subgroups $\Gamma_{0}(N)$ with non-trivial Dirichlet characters, and in and 64, ch. 3] we also considered general subgroups of the modular group. Recently, in [6], this algorithm, in combination with other theoretical methods, was used to show the existence of certain Maass waveforms close to the tentative waveforms produced by the algorithm.

The aim of the present paper (which is based on [64, ch. 2]) is to demonstrate how to extend the algorithm to general multiplier systems and arbitrary real weights.

The first section contains a brief review of the basic theory of multiplier systems; then we will introduce the notion of Maass waveforms in this context. We will also provide some details on the different operators that act on the space of Maass waveforms with a non-trivial multiplier system. While being of interest in themselves, these operators can also be used in combination with other tests of reliability and accuracy of the algorithm.

In Section 7 we will give the specifics of how the algorithm is modified, and in the last section we present a selection of the results which has been obtained with the described method.

1.2. Summary of notation. We will use the notation $e(x)=e^{2 \pi i x}$, and for a complex number $z$ we always use the principal branch of the argument, $-\pi<$ $\operatorname{Arg} z \leq \pi$

Let $\mathcal{H}=\{z=x+i y \mid y>0\}$ be the upper half-plane equipped with the hyperbolic line- and area-elements $d s^{2}=\frac{|d z|^{2}}{y^{2}}$ and $d \mu=\frac{d x d y}{y^{2}}$ respectively. The boundary of $\mathcal{H}$ is $\partial \mathcal{H}=\mathbb{R} \cup\{\infty\}$. The isometry group of $\mathcal{H}$ is identified with 
$P G L(2, \mathbb{R})=G L(2, \mathbb{R}) /\{ \pm \mathrm{Id}\}$, where $G L(2, \mathbb{R})$ is the group of invertible two-bytwo matrices with real elements and $\operatorname{Id}=\left(\begin{array}{ll}1 & 0 \\ 0 & 1\end{array}\right)$. For $\gamma=\left(\begin{array}{ll}a & b \\ c & d\end{array}\right) \in G L(2, \mathbb{R})$ and $z \in \mathcal{H}$ we define an action by

$$
\gamma z= \begin{cases}\frac{a z+b}{c z+d}, & \text { if } \quad a d-b c>0, \\ \frac{a \bar{z}+b}{c \bar{z}+d}, & \text { if } \quad a d-b c<0 .\end{cases}
$$

The subgroup of orientation-preserving isometries of $\mathcal{H}$ is given by $\operatorname{PSL}(2, \mathbb{R})=$ $S L(2, \mathbb{R}) /\{ \pm \operatorname{Id}\}$, where $S L(2, \mathbb{R})$ is the subgroup of $G L(2, \mathbb{R})$ consisting of matrices with determinant 1 . For any subgroup $\Gamma \subseteq P G L(2, \mathbb{R})$, we use $\bar{\Gamma}$ to denote the inverse image of $\Gamma$ in $G L(2, \mathbb{R})$. Note that this forces $-\operatorname{Id} \in \bar{\Gamma}$. We are mainly interested in Fuchsian groups, i.e. discrete subgroups of $\operatorname{PSL}(2, \mathbb{R})$. Of particular interest is the subgroup consisting of matrices with integer entries, the modular group, $P S L(2, \mathbb{Z})$. We are also interested in the so-called Hecke congruence subgroups, $\Gamma_{0}(N)=\left\{\left(\begin{array}{ll}a & b \\ c & d\end{array}\right) \in P S L(2, \mathbb{Z}) \mid c \equiv 0 \bmod N\right\}$, defined for any positive integer $N$ (note that $\Gamma_{0}(1)=P S L(2, \mathbb{Z})$ ).

We say that an element $\gamma$ of $P S L(2, \mathbb{R})$ is elliptic, parabolic or hyperbolic if the absolute value of the trace of the associated matrix is smaller than, equal to or greater than 2 respectively, or equivalently, if $\gamma$ has one fixed point in $\mathcal{H}$, one (double) fixed point in $\partial \mathcal{H}$ or two (different) fixed points in $\partial \mathcal{H}$. Fixed points of parabolic elements are called cusps.

If $\Gamma \subset P S L(2, \mathbb{R})$ is a finitely generated Fuchsian group, we identify the set of $\Gamma$-orbits with a connected subset of $\mathcal{H}, \mathcal{F}=\Gamma \backslash \mathcal{H}$, a fundamental domain of $\Gamma$. If $\Gamma$ has a set $p_{1}, \ldots, p_{\kappa}$ of inequivalent cusps, then the set $\mathcal{F}$ will meet $\partial \mathcal{H}$ at $\kappa$ inequivalent points which we will also denote by $p_{1}, \ldots, p_{\kappa}$, and we usually abuse the notation and call these points the cusps of $\Gamma$ (or of $\mathcal{F}$ ). By conjugation we may always assume $p_{1}=i \infty$. Corresponding to a cusp $p_{j}$ of $\Gamma$ we use $S_{j}$ to denote a parabolic generator of $\Gamma_{p_{j}}$ - the subgroup of $\Gamma$ which fixes $p_{j}$. We also choose a cusp normalizer, $\sigma_{j} \in P S L(2, \mathbb{R})$, with the property that $\sigma_{j}(\infty)=p_{j}$ and $\sigma_{j} S \sigma_{j}^{-1}=S_{j}$, where $S=\left(\begin{array}{ll}1 & 1 \\ 0 & 1\end{array}\right)$ is the parabolic generator of $\operatorname{PSL}(2, \mathbb{Z})$ (the other generator being $\left.T=\left(\begin{array}{cc}0 & -1 \\ 1 & 0\end{array}\right)\right)$. The map $\sigma_{j}$ is uniquely determined up to a translation $S^{k}$. If $\mathcal{F}$ meet $\partial \mathcal{H}$ at the points $q_{i}, 1 \leq j \leq \kappa_{0}$ we fix a set of maps $U_{i} \in \Gamma$ such that $U_{i} q_{i}=p_{j}$, where $p_{j}$ is the unique cusp equivalent to $q_{i}$.

\section{Multiplier SYStems}

2.1. Introduction. We will give a brief introduction to multiplier systems; for more extensive treatments see [22, pp. 331-338], [53, or [55, pp. 70-87].

Let $\Gamma$ be a Fuchsian group and $m$ an even integer. Classically, a function $\varphi$, meromorphic on the upper half-plane $\mathcal{H}$, which satisfies

$$
\varphi(A z)=\Theta_{A}(z ; m) \varphi(z)=(c z+d)^{m} \varphi(z), \quad \forall A=\left(\begin{array}{cc}
a & b \\
c & d
\end{array}\right) \in \Gamma,
$$

is called an automorphic form of weight $m$ for $\Gamma$. The function

$$
\Theta_{A}(z ; m)=(c z+d)^{m}, A=\left(\begin{array}{cc}
a & b \\
c & d
\end{array}\right) \in \Gamma
$$


is said to be an automorphy factor on $\Gamma$. The classical theory of automorphic forms is well-known; for instance, if $m=2$, then the automorphic forms can be identified with the meromorphic differential forms of degree 1 on the orbifold (classical Riemann surface) $\Gamma \backslash \mathcal{H}$.

We observe that, for even $m$, the number $(c z+d)^{m}$ is uniquely defined and the automorphy factor $\Theta_{A}(z ; m)$ in (2.1) clearly satisfies

$$
\Theta_{A}(B z ; m) \Theta_{B}(z ; m)=\Theta_{A B}(z ; m) .
$$

To generalize these notions to arbitrary real $m$, there needs to be a choice of branch of the argument, and to make certain everything is well-defined, we have to introduce the notion of a multiplier system.

Definition 1. For any real number $m$ define

$j_{A}(z ; m)=e^{i m \operatorname{Arg}(c z+d)}=\frac{(c z+d)^{m}}{|c z+d|^{m}}=\left(\frac{c z+d}{c \bar{z}+d}\right)^{\frac{m}{2}}, A=\left(\begin{array}{cc}a & b \\ c & d\end{array}\right) \in S L(2, \mathbb{R})$,

where $\operatorname{Arg} z$ denotes the principal branch of the $\operatorname{argument},-\pi<\operatorname{Arg} z \leq \pi$. To adapt the relation $(*)$, we also write

$$
\sigma_{m}(A, B)=j_{A}(B z ; m) j_{B}(z ; m) j_{A B}(z ; m)^{-1} .
$$

It is clear that for integer $m, \sigma_{m}(A, B)=1$, but it can also be shown (cf. [53, $\S 2$, pp. 42-50]) that the only values which $\sigma_{m}$ can take are 1 and $e^{ \pm 2 \pi i m}$.

Definition 2. $v: \bar{\Gamma} \rightarrow S^{1}=\{z|| z \mid=1\}$ is said to be a multiplier system of weight $m$ on $\bar{\Gamma}$ if

- $v(-I)=e^{-\pi i m}$, and

- $v(A B)=\sigma_{m}(A, B) v(A) v(B), \forall A, B \in \bar{\Gamma}$.

Observe that $v$ can be regarded equally well as a multiplier system of any weight $m^{\prime} \equiv m \bmod 2$. The question of whether there exist multiplier systems of a given weight and on a given group is most easily answered by the following proposition (cf. [22, Prop. 2.1, p. 333]).

Proposition 1. We are given $v: \bar{\Gamma} \rightarrow S^{1}$ and $m \in \mathbb{R}$. The following are equivalent:

- $v(T)$ is a multiplier system of weight $m$ on $\bar{\Gamma}$.

- There exists a function $\varphi \not \equiv 0$ on $\mathcal{H}$ which is either $C^{\infty}$ or meromorphic such that

$$
\varphi(A z)=v(A) \varphi(z)(c z+d)^{m}, \forall A=\left(\begin{array}{ll}
a & b \\
c & d
\end{array}\right) \in \bar{\Gamma} .
$$

A $\varphi$ as above is said to be an automorphic form of weight $m$ and a multiplier system $v$ on $\Gamma$.

Definition 3. Given a multiplier system $v$ on $\bar{\Gamma}$ and an element $\alpha \in G L(2, \mathbb{R})$ we define a multiplier system, $v^{\alpha}$, on the group $\alpha^{-1} \bar{\Gamma} \alpha$ by

$$
v^{\alpha}(A)=v\left(\alpha A \alpha^{-1}\right) \frac{\sigma_{m}\left(\alpha A \alpha^{-1}, \alpha\right)}{\sigma_{m}(\alpha, A)}, A \in \alpha^{-1} \bar{\Gamma} \alpha .
$$

That this indeed gives a multiplier system on $\alpha^{-1} \bar{\Gamma} \alpha$ is shown in [42, p. 138].

Using Proposition 1, we will construct the two most widely used multiplier systems in the following sections. Compare [22, pp. 334-337]. 


\subsection{The $\eta$ multiplier system.}

2.2.1. The $\eta$-function. The Dedekind $\eta$-function is a holomorphic function on $\mathcal{H}$, defined by

$$
\eta(z)=e\left(\frac{z}{24}\right) \prod_{n=1}^{\infty}(1-e(n z)) .
$$

It is clear from the definition that $\eta(z) \neq 0$ for $z \in \mathcal{H}$ and that, for each $k \in \mathbb{R}$, $\eta^{2 k}$ can be defined as a holomorphic function on $\mathcal{H}$. (Cf. [55, p. 205].) Note that $\eta(z)^{24}$ is the famous discriminant function $\Delta(z)$. Cf. [55, pp. 196-197]. It is clear that $\eta(z+1)=e\left(\frac{1}{24}\right) \eta(z)$, and as for $\Delta(z)$ it is also possible to express $\eta(z)$ as a lacunary Fourier series (cf. [46, p. 18]).

2.2.2. The multiplier system. It can be proved (cf. Thm. 3.1 and Thm. 3.4 [1, p. 48 and p. 52]) that $\eta$ satisfies the following functional equations:

$$
\begin{aligned}
\eta\left(\frac{-1}{z}\right) & =(-i z)^{\frac{1}{2}} \eta(z), \text { and in general, } \\
\eta(A z) & =v_{\eta}(A)(c z+d)^{\frac{1}{2}} \eta(z), \forall A=\left(\begin{array}{ll}
a & b \\
c & d
\end{array}\right) \in S L(2, \mathbb{Z}),
\end{aligned}
$$

where $v_{\eta}$ is defined below. This functional equation expresses the fact that $\eta$ is an $S L(2, \mathbb{Z})$-automorphic form of weight $\frac{1}{2}$ and multiplier system given by $v_{\eta}$. Accordingly the function $\eta^{2 k}$ is an $S L(2, \mathbb{Z})$-automorphic form of weight $k$ and multiplier system given by $v_{\eta}^{2 k}$, and we can use $\eta^{2 k}$ in the context of Proposition 1 to assure the existence of the multiplier system, $v_{\eta, k}=v_{\eta}^{2 k}$, of weight $k$ on $S L(2, \mathbb{Z}$ ) (and any of its subgroups e.g. $\left.\Gamma_{0}(N)\right)$. We have the following explicit formula for $v=v_{\eta}^{2 k}$ :

$$
\frac{1}{2 \pi i} \log v\left(\left(\begin{array}{ll}
a & b \\
c & d
\end{array}\right)\right)=\left\{\begin{array}{cc}
\frac{k b}{12}, & a=d=1, c=0, \\
k\left(\frac{a+d-3 c}{12 c}-s(d, c)\right), & c>0,
\end{array}\right.
$$

and for $c<0$ we use the fact that $v(-A)=e^{-k \pi i} v(A)$ (cf. Definition 2). Here $s(d, c)$ is the Dedekind sum,

$$
s(d, c)=\sum_{n=1}^{c-1} \frac{n}{c}\left(\left(\frac{d n}{c}\right)\right),
$$

where $((x))$ is the saw-tooth function

$$
((x))= \begin{cases}x-\lfloor x\rfloor-\frac{1}{2}, & \text { if } x \notin \mathbb{Z}, \\ 0, & \text { if } x \in \mathbb{Z},\end{cases}
$$

and $\lfloor x\rfloor$ is the greatest integer less than or equal to $x$. Note that if $x$ is not an integer, then $\lfloor-x\rfloor=-\lfloor x\rfloor-1$ so $((-x))=-((x))$, and hence $s(-d, c)=-s(d, c)$ if $\operatorname{gcd}(d, c)=1$.

Remark 1 . It is also possible to express the $\eta$ multiplier explicitly without Dedekind sums but using extended quadratic residue symbols instead. We have the following formulas from Knopp [31, p. 51] or van Lint [71, Thm. 3]:

$$
\begin{aligned}
& v_{\eta}\left(\left(\begin{array}{ll}
a & b \\
c & d
\end{array}\right)\right) \\
& \quad= \begin{cases}\left(\frac{c}{d}\right) e\left(\frac{1}{24}\left[(a+d) c-b d\left(c^{2}-1\right)+3 d-3-3 c d\right]\right), & c>0, \text { even, } \\
\left(\frac{d}{c}\right) e\left(\frac{1}{24}\left[(a+d) c-b d\left(c^{2}-1\right)-3 c\right]\right), & c>0, \text { odd. }\end{cases}
\end{aligned}
$$


(Note that the symbols $\left(\frac{c}{d}\right)_{*}$ and $\left(\frac{d}{c}\right)^{*}$ of [31, 71] agree with our symbols in these two cases.)

Remark 2. It is known that, for each $k \in \mathbb{R}$, there exist exactly 6 different multiplier systems of weight $k$ on $P S L(2, \mathbb{Z})$ (cf. [55, $\S 3.4$, pp. 83, 206] or [42, Thm. 19, p. 132]). We will denote these by $v_{\eta, k}^{(r)}=v_{\eta}^{2(k+r)}$, where $r \in\{0,2,4,6,8,10\}$. Compare [55, eq. (6.4.7)]; one knows, of course, that $v_{\eta}^{24}=1$.

When dealing with the modular group and weight $k$, it is sufficient to consider only the multiplier system $v_{\eta, k}^{(0)}=v_{\eta, k}=v_{\eta}^{2 k}$ (for reasons to be discussed later in Section 4.4.1).

2.3. The $\theta$ multiplier system. On any subgroup of $P S L(2, \mathbb{Z})$ we can always use the $\eta$-multiplier system, but in general, on subgroups of $P S L(2, \mathbb{Z})$ there are also other multiplier systems available. In particular, on $\Gamma_{0}(4)$ there is a multiplier system of weight $\frac{1}{2}$ which is interesting from an arithmetical point of view.

It is well-known (cf. [60] or [28]) that the Jacobi theta function

$$
\theta(z)=\sum_{-\infty}^{\infty} e\left(n^{2} z\right), z \in \mathcal{H},
$$

is automorphic on $\Gamma_{0}(4)$ with weight $k=\frac{1}{2}$ and can be used to define a multiplier system on $\Gamma_{0}(4)$. Using the Poisson summation formula one can prove (cf. [18, pp. 72-75] or [28, pp. 167-168]) that the theta function satisfies

$$
\theta\left(\frac{-1}{2 z}\right)=(-i z)^{\frac{1}{2}} \theta\left(\frac{z}{2}\right),
$$

and one can also prove the general formula (cf. [28, Thm. 10.10, p. 177] or [60, p. 447]):

$$
\theta(A z)=v_{\theta}(A)(c z+d)^{\frac{1}{2}} \theta(z), A=\left(\begin{array}{cc}
a & b \\
c & d
\end{array}\right) \in \Gamma_{0}(4) .
$$

The multiplier $v_{\theta}(A)$ can be expressed explicitly as

$$
v_{\theta}(A)=\bar{\epsilon}_{d}\left(\frac{c}{d}\right)
$$

where $\epsilon_{d}=1$ if $d \equiv 1 \bmod 4$ and $\epsilon_{d}=i$ if $d \equiv-1 \bmod 4$, and $\left(\frac{c}{d}\right)$ denotes the extended quadratic residue symbol defined as the traditional Jacobi symbol if $0<d \equiv 1 \bmod 2$ and extended by

$$
\left(\frac{c}{d}\right)=\frac{c}{|c|}\left(\frac{c}{-d}\right), c \neq 0
$$

and

$$
\left(\frac{0}{d}\right)= \begin{cases}1 & \text { if } d= \pm 1 \\ 0 & \text { otherwise }\end{cases}
$$

For the sake of completeness we also use the traditional Kronecker extension, i.e. we define

$$
\left(\frac{c}{2}\right)=\left(\frac{2}{c}\right)
$$

One can verify that our symbol $(\vdots)$ satisfies reciprocity relations similar to the usual ones: 
Proposition 2. Suppose that $c, d \in \mathbb{Z}$ are odd and $c \neq 0$. Then we have:

$$
\begin{aligned}
\left(\frac{-1}{d}\right) & =(-1)^{\left(\frac{d-1}{2}\right)}, \\
\left(\frac{c}{d}\right) & = \begin{cases}\left(\frac{d}{c}\right)(-1)^{\left(\frac{d-1}{2}\right)\left(\frac{c-1}{2}\right)}, & d, \text { or } c>0, \\
-\left(\frac{d}{c}\right)(-1)^{\left(\frac{d+1}{2}\right)\left(\frac{c+1}{2}\right)}, & d, \text { and } c<0 .\end{cases}
\end{aligned}
$$

Remark 3. Relations (2.6) and (2.7) can also be proved using the corresponding relations (2.3) for $\eta$ and the following relation between the $\eta$ and the $\theta$ functions (cf. [28, p. 177] or [31, Thm. 12, p. 46]): $\theta(z)=\frac{\eta\left(\frac{z+1}{2}\right)}{\eta(z+1)}$.

\section{MAASS WAVEFORMS}

From now on we consider arbitrary $k \in \mathbb{R}$. The slash-operator $f_{\mid A}(z)=f(A z)$ can be extended to an operator of weight $k$ as

$$
f_{[[k, A]}(z)=f(A z) j_{A}(z ; k)^{-1},
$$

and the natural analog of the Laplace-Beltrami operator, $\Delta$, which is invariant under this action is the weight- $k$ Laplacian:

$$
\Delta_{k}=\Delta-i y k \frac{\partial}{\partial x}=y^{2}\left(\frac{\partial^{2}}{\partial x^{2}}+\frac{\partial^{2}}{\partial y^{2}}\right)-i y k \frac{\partial}{\partial x} .
$$

If $\Gamma$ is a Fuchsian group we define the space $\mathcal{M}(\Gamma, v, k, \lambda)$ consisting of Maass waveforms on $\Gamma$, of weight $k$, multiplier system $v$ and eigenvalue $\lambda$, as the space of functions which satisfy the following conditions:

$$
\begin{aligned}
& f_{\mid[A, k]}(z)=v(A) f(z), \forall A=\left(\begin{array}{ll}
a & b \\
c & d
\end{array}\right) \in \bar{\Gamma}, \\
& \Delta_{k} f+\lambda f=0, \text { and } \\
& \int_{\mathcal{F}}|f|^{2} d \mu<\infty .
\end{aligned}
$$

Observe that condition 1) is equivalent to

$$
f(A z)=v(A) j_{A}(z ; k) f(z), \forall A=\left(\begin{array}{cc}
a & b \\
c & d
\end{array}\right) \in \bar{\Gamma} .
$$

For purposes of the computational work to be described in this paper, we shall be content to restrict ourselves to cases where $\lambda>\frac{1}{4}$. (Cf. also paragraph 4 of section 8.1 below.)

Instead of the Bessel equation in the case of weight 0, condition 2) above gives us the Whittaker equation, and using the method of separation of variables gives us Whittaker functions instead of the K-Bessel functions at weight 0 (for complete details see [22, Chap. 9]). Since $f(x+i y)$ is no longer periodic in $x$, but instead satisfies $f(z+1)=v(S) f(z)=e(\alpha) f(z)$, with $\alpha \in[0,1)$, the Fourier series of $f$ can ([22, pp. 26,348,420(19)]) be written as

$$
f(z)=\sum_{\substack{-\infty \\ n+\alpha \neq 0}}^{\infty} \frac{c(n)}{\sqrt{|n+\alpha|}} W_{\operatorname{sgn}(n+\alpha) \frac{k}{2}, i R}(4 \pi|n+\alpha| y) e((n+\alpha) x),
$$

where $W_{l, \mu}(x)$ is the Whittaker function in standard notation (cf. [15, vol. I, p. 264]) and $R$ is the usual spectral parameter, $\lambda=\frac{1}{4}+R^{2}$. One notes here that $W_{0, i R}(x)=\pi^{-\frac{1}{2}} x^{\frac{1}{2}} K_{i R}\left(\frac{x}{2}\right)$. For $k=0$, the expansion above thus reduces to the usual Fourier expansion with $2 y^{\frac{1}{2}} K_{i R}(2 \pi|n+\alpha| y)$ as in [22, p. 26, prop. 4.12]. 
If we have more than one cusp we define functions $f_{j}$ related to $f$ at each cusp, $p_{j}$, of $\Gamma$ by using the cusp normalizing maps $\sigma_{j}$ from Section 1.2 and setting $f_{j}(z)=$ $f_{\mid\left[\sigma_{j}, k\right]}(z)=j_{\sigma_{j}}(z ; k)^{-1} f\left(\sigma_{j} z\right)$. It is easy to see that

$$
f_{j}(z+1)=v\left(S_{j}\right) f_{j}(z)=e\left(\alpha_{j}\right) f_{j}(z)
$$

with $\alpha_{j} \in[0,1)$ (cf. [28, p. 41]). Thus the Fourier series of $f$ at the cusp $j$ can be written as

$$
f_{j}(z)=\sum_{n=-\infty}^{\infty} \frac{c_{j}(n)}{\sqrt{\left|n+\alpha_{j}\right|}} W_{s g n\left(n+\alpha_{j}\right) \frac{k}{2}, i R}\left(4 \pi\left|n+\alpha_{j}\right| y\right) e\left(\left(n+\alpha_{j}\right) x\right)
$$

As in the case of weight 0 and Dirichlet character, we say that the cusp number $j$ is open or singular if $\alpha_{j}=0$ and closed if $\alpha_{j} \neq 0$. If all cusps of $\Gamma$ are singular for the multiplier system $v$, we say that $v$ is a singular multiplier system for $\Gamma$.

Remark 4. Observe that, for the eta-multiplier on $\operatorname{PSL}(2, \mathbb{Z})$ and weight $k$, we have $\alpha=\alpha_{1}=\frac{k}{12}$.

3.1. Decomposition of the discrete spectrum. It is known (see for example [11] or [22]) that closed cusps (i.e. $v\left(S_{j}\right) \neq 1$ ) do not contribute to the continuous spectrum, and if all cusps are closed there is only the discrete part of the spectrum left, and this is spanned by the Maass waveforms. We also know (see [22, p. 385]) that on the modular group with weight $k$ the smallest eigenvalue is

$$
\lambda_{\min }=\frac{|k|}{2}\left(1-\frac{|k|}{2}\right)
$$

or larger. In the case of $P S L(2, \mathbb{Z})$ and $k \geq 0, F(z)=y^{\frac{k}{2}} \eta(z)^{2 k}$ has eigenvalue equal to $\lambda_{\min }$.

In this paper, any eigenvalues $\lambda \in\left[\lambda_{\min }, \frac{1}{4}\right]$ will be regarded as exceptional. The non-exceptional eigenvalues thus satisfy $\frac{1}{4}<\lambda_{0} \leq \lambda_{1} \leq \cdots \leq \lambda_{n} \rightarrow \infty$. One can obtain lower bounds for the eigenvalue $\lambda_{0}$ (see [11, p. 183]), but in light of the numerical experiments in Section 8.1 they are not very effective (cf. Figure 21).

\section{Operators}

4.1. Conjugation and reflection. Let $J$ and $K$ denote the reflection, $J z=-\bar{z}$, and conjugation, $K z=\bar{z}$. Then $J$ and $K$ act as involutions on the space of Maass waveforms via the operations

$$
\begin{aligned}
K f & =f_{\mid K}(z)=\overline{f(z),} \\
J f & =f_{\mid J}(z)=f(-\bar{z}) .
\end{aligned}
$$

It follows from the definition of the action of $G L(2, \mathbb{R})$ on $\mathcal{H}$ (cf. page 2376) that we can use the matrix $J=\left(\begin{array}{cc}1 & 0 \\ 0 & -1\end{array}\right)$ in $G L(2, \mathbb{R})$ to represent the operator $J$. For $A=\left(\begin{array}{ll}a & b \\ c & d\end{array}\right)$ we define

$$
A^{*}=J A J^{-1}=\left(\begin{array}{cc}
a & -b \\
-c & d
\end{array}\right),
$$

and then $A^{* *}=A$, and $A(z)_{\mid K}=-A^{*}(z)$, also meaning that $-\overline{A(z)}=A^{*}(-\bar{z})$.

Remark 5. It is easy to verify that if $f \in \mathcal{M}\left(\Gamma_{0}(N), v, k, \lambda\right)$, then

$$
K f \in \mathcal{M}\left(\Gamma_{0}(N), \bar{v},-k, \lambda\right) \text { and } \quad J f \in \mathcal{M}\left(\Gamma_{0}(N), v^{*},-k, \lambda\right) \text {, }
$$


where $v^{*}$ is the multiplier system determined by

$$
v^{*}(A)=v\left(A^{*}\right) \cdot\left\{\begin{array}{ll}
1, & c \neq 0, \\
e^{\pi i k(1-\operatorname{sgn}(d))}, & c=0,
\end{array}, \text { for } A=\left(\begin{array}{ll}
a & b \\
c & d
\end{array}\right) .\right.
$$

Of particular interest is the involution obtained by combining $J$ and $K$, i.e.

$$
K J f(z)=f_{\mid J K}(z)=\overline{f(-\bar{z})} .
$$

It is easily seen that if $f$ has Fourier coefficients $c_{j}(n)$, then (by [15, p. 265(8)]) $f_{\mid J K}$ has Fourier coefficients $\overline{c_{j}(n)}$, and we thus would like to have $f$ and $f_{\mid J K}$ belonging to the same space (i.e. transform according to the same multiplier system), since then we can assume that the Fourier coefficients are real.

It is clear that if $f \in \mathcal{M}\left(\Gamma_{0}(N), v, k, \lambda\right)$, then $K J f \in \mathcal{M}\left(\Gamma_{0}(N), \overline{v^{*}}, k, \lambda\right)$, so we are left to see whether or not $\overline{v^{*}}=v$. By using the explicit formulas one can verify that indeed $\overline{v^{*}}=v$ for both $v_{\theta}$ and $v_{\eta}$ (see [64, pp. 66-68] for details), and we arrive at the following proposition.

Proposition 3. If $v$ is either the $\eta$ - or the $\theta$-multiplier system (in the latter case $4 \mid N)$, then a basis $\left\{g_{1}, \ldots, g_{m}\right\}$ of $\mathcal{M}\left(\Gamma_{0}(N), v, k, \lambda\right)$ can be chosen so that each $g_{j}$ can be expanded in a Fourier series at $\infty$ with real coefficients.

Proof. We have seen that for both the theta and the eta multiplier systems the product $K J$ is a conjugate-linear involution of the space $\mathcal{M}\left(\Gamma_{0}(N), v, k, \lambda\right)$, and hence we can assume that any $f \in \mathcal{M}\left(\Gamma_{0}(N), v, k, \lambda\right)$ is an eigenfunction of $K J$ with eigenvalue $\epsilon$, where $|\epsilon|=1$. Note that if $f(z)$ has a Fourier series expansion as above with Fourier coefficients $c(n)$, then $f_{\mid K J}$ has Fourier coefficients $\overline{c(n)}$ and hence $\overline{c(n)}=\epsilon c(n)$. Finally we observe that if $\epsilon=e^{i \theta}$ we can look at the function $g=e^{i \frac{\theta}{2}} f$ which then satisfies $K J g=e^{-i \frac{\theta}{2}} K J f=e^{-i \frac{\theta}{2}} e^{i \theta} f=g$. After proper normalization it is thus no restriction to assume that the eigenvalue of $K J$ is $\epsilon=1$, and that the Fourier coefficients are real.

Remark 6 . For the sake of completeness it should be remarked that in general one cannot simultaneously take Fourier coefficients at cusps other than $\infty$ to be real (cf. the next subsection where we introduce the map $\omega_{N}$, which is a cusp normalizing map for the cusp at 0 and which has eigenvalues $\left.\pm i^{-k}\right)$.

4.2. The involution $\tau_{N}$. As in the case of zero weight (e.g. 65]) we define $\omega_{N} z=$ $\frac{-1}{N z}$, or equivalently $\omega_{N}=\left(\begin{array}{cc}0 & \frac{-1}{\sqrt{N}} \\ \sqrt{N} & 0\end{array}\right)$. We know that $\omega_{N}$ is an involution of $\Gamma_{0}(N)$, i.e. $\Gamma_{0}(N)=\omega_{N} \Gamma_{0}(N) \omega_{n}^{-1}$, but the question is how it relates to the weight and multiplier system.

If $f \in \mathcal{M}\left(\Gamma_{0}(N), v, k, \lambda\right)$ it is easy to see that $f_{\mid\left[k, \omega_{N}\right]} \in \mathcal{M}\left(\Gamma_{0}(N), v^{\omega_{N}}, k, \lambda\right)$, and it is also easy to verify that $v^{\omega_{N}}(T)=v\left(\omega_{N} T \omega_{N}^{-1}\right)$. We also have

$$
\begin{aligned}
f_{\left|\left[k, \omega_{N}\right]\right|\left[k, \omega_{N}\right]}(z) & =j_{\omega_{N}}(z ; k)^{-1} f\left(\omega_{N} z\right)_{\mid\left[k, \omega_{N}\right]}=j_{\omega_{N}}(z ; k)^{-1} j_{\omega_{N}}\left(\omega_{N} z ; k\right)^{-1} f\left(\omega_{N}^{2} z\right) \\
& =e^{-i k \operatorname{Arg}(\sqrt{N} z)} e^{-i k \operatorname{Arg}(-1 / \sqrt{N} z)} f(z)=e^{-i \pi k} f(z),
\end{aligned}
$$

and hence if we define the operator $\tau_{N}$ by

we have that

$$
\tau_{N} f(z)=e^{i k \frac{\pi}{2}} f_{\mid\left[k, \omega_{N}\right]}(z),
$$

$$
\tau_{N}^{2}=\mathrm{Id}
$$


To show that $\tau_{N}$ is a linear involution, we also have to verify that $v^{\omega_{N}}=v$. This is easily done in the two cases $N=1$ together with $v=v_{\eta}$ and $N=4$ together with $v=v_{\theta}$. Therefore, we conclude:

Proposition 4. If $N=1$ and $v=v_{\eta}$, or $N=4$ and $v=v_{\theta}$, the operator:

$$
\tau_{N}: \mathcal{M}\left(\Gamma_{0}(N), v, k, \lambda\right) \rightarrow \mathcal{M}\left(\Gamma_{0}(N), v, k, \lambda\right),
$$

defined by

$$
\tau_{N} f(z)=e^{i k \frac{\pi}{2}} f_{\left[\left[k, \omega_{N}\right]\right.}=e^{-i k\left(\operatorname{Arg} z-\frac{\pi}{2}\right)} f\left(\frac{-1}{N z}\right),
$$

is a linear involution, i.e. $\tau_{N}(a f)=a \tau_{N} f$ for all $a \in \mathbb{C}$ and $\tau_{N}^{2} f=f$. Hence it has eigenvalues \pm 1 .

Remark 7. Note that in the case of $\Gamma_{0}(4)$ (i.e. $\left.N=4\right)$ if $\tau_{N} f(z)= \pm f(z)$, then

$$
f_{2}=f_{\mid \omega_{N}}=e^{-i k \frac{\pi}{2}} \tau_{N} f= \pm e^{-i k \frac{\pi}{2}} f
$$

which means that the Fourier coefficients at the cusp at 0 are proportional to the coefficients at $i \infty$ :

$$
c_{2}(n)= \pm e^{-i \frac{\pi}{2} k} c_{1}(n)= \pm i^{-k} c_{1}(n)
$$

\subsection{The operator $L$.}

Definition 4. For $N=4$ and the $\theta$-multiplier system at weight $\frac{1}{2}$, following Kohnen [32] or Katok-Sarnak [29] we define the operator $L$ acting on $\mathcal{M}\left(\Gamma_{0}(4), v_{\theta}, \frac{1}{2}, R\right)$ by

$$
L=\frac{1}{\sqrt{2}} \tau_{4} T_{4, \frac{1}{2}}^{v_{\theta}}
$$

where $T_{4, \frac{1}{2}}^{v_{\theta}}$ is the following (exceptional) Hecke operator:

$$
T_{4, \frac{1}{2}}^{v_{\theta}} f(z)=\frac{1}{2} \sum_{j \bmod 4} f\left(\frac{z+j}{4}\right)
$$

Cf. Section 4.5.1 It can be shown that the operator $L$ is self-adjoint, commutes with $\Delta_{\frac{1}{2}}$ and all Hecke operators $T_{p^{2}, \frac{1}{2}}^{v_{\theta}}, p \neq 2$ (cf. (4.9)) and has the eigenvalues 1 and $-\frac{1}{2}$. Cf. 32 and [48. Suppose that $f(z)$ has Fourier expansions of (3.2) at the cusps $p_{1}=\infty, p_{2}=0$ and $p_{3}=-\frac{1}{2}$ with Fourier coefficients $a_{j}(n)$ respectively. By calculations similar to 32 or 32 , 5] it can be shown that $L f$ has Fourier coefficients $b(n)$ (with respect to $\infty$ ) given by

$$
b(n)=\frac{1}{2} \begin{cases}a_{1}(n)+(1+i) a_{2}\left(\frac{n}{4}\right), & n \equiv 0 \bmod 4 \\ a_{1}(n)+\sqrt{2} a_{3}\left(\frac{n-1}{4}\right)(-1)^{\frac{n-1}{4}}, & n \equiv 1 \bmod 4 \\ -a_{1}(n), & n \equiv 2,3 \bmod 4 .\end{cases}
$$

Let $V^{+} \subseteq \mathcal{M}\left(\Gamma_{0}(4), v_{\theta}, \frac{1}{2}, \lambda\right)$ denote the subspace introduced by Kohnen in [32, i.e. $V^{+}$consists of all $f$ with Fourier coefficients $a_{1}(n)=0$ for $n \equiv 2,3 \bmod 4$. By using (4.3) it is easy to verify that $V^{+}$is exactly the eigenspace of $L$ corresponding to the eigenvalue 1 . Unfortunately the eigenspace $V^{-}$corresponding to the eigenvalue $-\frac{1}{2}$ is not as simple to characterize. However, (4.3) can be used to identify $V^{-}$by means of certain relations between coefficients at the cusps at $\infty, 0$ and $-\frac{1}{2}$. E.g. if $a_{1}(1)=1$, then $a_{3}(0)=-\sqrt{2}$, and if $a_{1}(1)=0$, then $a_{3}(0)=0$. 
Clearly $T_{4, \frac{1}{2}}^{v_{\theta}}$ does not in general commute with $L$, but in the case $L f=\lambda f$, $T_{4, \frac{1}{2}} f=\lambda_{4} f$ and $\tau_{N} f=\epsilon f$ (with $\epsilon= \pm 1$ ), then $a_{1}(4)=\epsilon \sqrt{2} \lambda=-\frac{\epsilon}{\sqrt{2}}, \sqrt{2} \epsilon$. This should be compared with the results on newforms at weight zero, e.g. [3, p. 147] and [64, p. 31 ].

4.4. Maass operators. So far, the operators we have seen act on spaces of Maass waveforms of a given weight and multiplier system.

We will show that we may limit the range of weights $k$ to investigate to $k \in[0,6]$. For this we will use the Maass lowering and raising operators, $E_{k}^{ \pm}$, which raise or lower the weight of a Maass waveform by units of 2 . They are defined by

$$
E_{k}^{ \pm}=i y \frac{\partial}{\partial x} \pm y \frac{\partial}{\partial y}+\frac{k}{2}
$$

and using the relation between the Whittaker function and the confluent hypergeometric function together with the transformation formulas [15, p. 258, (10)] (see also [42, p. 183 (middle)] and [43, p. 302 lines -3 and -1]), we see that for $k>0$ (here we set $Y=4 \pi|n+\alpha| y$ and $n_{\alpha}=n+\alpha$ )

$$
\begin{aligned}
& E_{k}^{+}\left(W_{\frac{k}{2}, i R}(Y) e\left(n_{\alpha} x\right)\right) \\
& =-W_{\frac{k+2}{2}, i R}(Y) e\left(n_{\alpha} x\right), \quad n_{\alpha}>0, \\
& E_{k}^{-}\left(W_{\frac{k}{2}, i R}(Y) e\left(n_{\alpha} x\right)\right) \\
& =-\left(\frac{k(k-2)}{4}+\frac{1}{4}+R^{2}\right) W_{\frac{k-2}{2}, i R}(Y) e\left(n_{\alpha} x\right), \quad n_{\alpha}>0, \\
& E_{k}^{+}\left(W_{-\frac{k}{2}, i R}(Y) e\left(n_{\alpha} x\right)\right) \\
& =\left(\frac{k(k+2)}{4}+\frac{1}{4}+R^{2}\right) W_{-\frac{k+2}{2}, i R}(Y) e\left(n_{\alpha} x\right), \quad n_{\alpha}<0, \\
& E_{k}^{-}\left(W_{-\frac{k}{2}, i R}(Y) e\left(n_{\alpha} x\right)\right) \\
& =W_{-\frac{k-2}{2}, i R}(Y) e\left(n_{\alpha} x\right), \quad n_{\alpha}<0 .
\end{aligned}
$$

To verify that they respect the weight $k$-action, it is easiest to proceed straight forward but to use the following form of the operators:

$$
\begin{aligned}
& E_{k}^{+}=(z-\bar{z}) \frac{\partial}{\partial z}+\frac{k}{2} \\
& E_{k}^{-}=-(z-\bar{z}) \frac{\partial}{\partial \bar{z}}+\frac{k}{2}
\end{aligned}
$$

and write $e^{-i \operatorname{Arg}(c z+d)}=\left(\frac{c \bar{z}+d}{c z+d}\right)^{\frac{1}{2}}$. Cf. [42, p. 178].

One can then show that $E_{k}^{ \pm}$maps $\mathcal{M}\left(\Gamma_{0}(N), v, k, \lambda\right)$ into $\mathcal{M}\left(\Gamma_{0}(N), v, k \pm 2, \lambda\right)$, and that the composition

$$
E_{k \pm 2}^{\mp} E_{k}^{ \pm}: \mathcal{M}\left(\Gamma_{0}(N), v, k, \lambda\right) \mapsto \mathcal{M}\left(\Gamma_{0}(N), v, k, \lambda\right)
$$

is just multiplication by a constant, which is readily seen to be non-zero anytime $\lambda>\frac{1}{4}$. Hence $E_{k}^{ \pm}$acts bijectively on the spaces corresponding to non-exceptional eigenvalues, i.e. they are always bijections for $\lambda>\frac{1}{4}$. 
4.4.1. Maass operators and the symmetry about $k=6$. First of all, observe that $E_{k}^{ \pm}$ only changes the weight and not the multiplier system $v$, but in view of the remark after Definition 2 it is clear that $v=v_{\eta}^{2 k}$ is also a multiplier system of weight $k+r$ for any $r \in 2 \mathbb{Z}$, and with the notation $v_{\eta, k}^{(r)}=v_{\eta}^{2(k+r)}$ it is clear that

$$
E_{k}^{+}: \mathcal{M}\left(\Gamma_{0}(N), v_{\eta, k}^{(r)}, k, \lambda\right) \rightarrow \mathcal{M}\left(\Gamma_{0}(N), v_{\eta, k+2}^{(r-2)}, k+2, \lambda\right)
$$

and

$$
E_{k}^{-}: \mathcal{M}\left(\Gamma_{0}(N), v_{\eta, k}^{(r)}, k, \lambda\right) \rightarrow \mathcal{M}\left(\Gamma_{0}(N), v_{\eta, k-2}^{(r+2)}, k-2, \lambda\right) .
$$

Our main purpose is to investigate the eigenvalues of Maass waveforms on the modular group when the weight and multiplier system are varied. That is, we would like to investigate the space $\mathcal{M}\left(\Gamma_{0}(1), v_{\eta, k}^{(r)}, k, \lambda\right)$ for all $k \in \mathbb{R}$ and $r \in$ $\{0,2,4,6,8,10\}$. Suppose that $\lambda>\frac{1}{4}$ so that the lowering and raising operators act bijectively. Then using $(k, r)$ to denote the space $\mathcal{M}\left(\Gamma_{0}(1), v_{\eta, k}^{(r)}, k, \lambda\right)$ and using $\approx$ to bijectively denote corresponding spaces we have:

- Since all $v_{\eta, k}^{(r)}$ are 24th-roots of unity we have trivially $(k, r+12)=(k, r)$.

- A composition of Maass operators which raises or lowers the weight by 12 will preserve the multiplier system. Hence $(k+12, r) \approx(k, r)$, and we may assume that $k \in[0,12]$.

- $K$ is a bijection from $(k, r)$ to $(-k,-r)=(-k, 12-r) \approx(12-k, 12-r)$, so there is no restriction to assume $k \in[0,6]$ and $r \in\{0,2,4,6,8,10\}$.

- By using the raising operator we see that $(k, 0) \approx(k+2,-2) \approx(k+2,10)$, and by repeated use we see that without loss of generality we can also assume $r=0$.

We are thus justified in our choice of restricting the investigation to the spaces $\mathcal{M}\left(\Gamma_{0}(1), v, k, \lambda\right)$ for $k \in[0,6]$ and $v=v_{\eta, k}^{(0)}=v_{\eta}^{2 k}$.

4.5. Hecke operators for non-trivial multiplier systems. We know that Hecke operators play an important role in the understanding of the theory of both modular forms and Maass waveforms at integer weights.

For general real weights, the Hecke operators are not important (and may well be non-existent), but we will begin with a general definition anyway, and then we will consider two special cases: $\Gamma_{0}(4)$ with the theta multiplier system and weight $\frac{1}{2}$, and $\Gamma_{0}(1)$ with the eta multiplier system and weight 1 .

The general introductory discussion is based on [67, but the specific example of integer weights (as worked out in detail in [66]) is based on ideas from [76] and 70 .

Let $\Gamma \subset P S L(2, \mathbb{R})$ be cofinite and suppose $v: \bar{\Gamma} \rightarrow S^{1}$ is a multiplier system of weight $k \in \mathbb{R}$. The commensurator of $\Gamma, \operatorname{comm}(\Gamma)$, in $\operatorname{PSL}(2, \mathbb{R})$ is defined as

$$
\operatorname{comm}(\Gamma)=\left\{\alpha \in P S L(2, \mathbb{R}) \mid \alpha \Gamma \alpha^{-1} \cap \Gamma \text { has finite index in } \Gamma \text { and } \alpha \Gamma \alpha^{-1}\right\} .
$$

We know that the Hecke operators are associated with the members of the commensurator, or actually with the double cosets, $\bar{\Gamma} \alpha \bar{\Gamma}$, for $\alpha \in \operatorname{comm}(\Gamma)$. Fix $\alpha \in \operatorname{comm}(\Gamma)$. It can be shown that if the multiplier system $v$ satisfies

$$
v(g)=v^{\alpha}(g), \forall g \in \bar{\Gamma} \cap \alpha^{-1} \bar{\Gamma} \alpha,
$$

then we can define an associated multiplier system, $v_{\alpha}$, on the double coset

$$
v_{\alpha}: \bar{\Gamma} \alpha \bar{\Gamma} \rightarrow S^{1}
$$


by setting

$$
v_{\alpha}\left(g_{1} \alpha g_{2}\right)=\sigma_{k}\left(g_{1} \alpha, g_{2}\right) \sigma_{k}\left(g_{1}, \alpha\right) v\left(g_{1}\right) v\left(g_{2}\right)
$$

for all $g_{1}, g_{2} \in \bar{\Gamma}$. It might be the case that there exists an associated multiplier system of $W=\chi v$, where $\chi$ is a character on $\bar{\Gamma} \alpha \bar{\Gamma}$, even though there does not exist an associated multiplier system of $v$ itself. Suppose that $v_{\alpha}$ exists as above, and that we have $\bar{\Gamma} \alpha \bar{\Gamma}=\bigcup_{i=1}^{d} \bar{\Gamma} \alpha_{i}$. We then define the Hecke operator $T_{\alpha, k}^{v}$ : $\mathcal{M}(\bar{\Gamma}, v, k, \lambda) \rightarrow \mathcal{M}(\bar{\Gamma}, v, k, \lambda)$ by

$$
\left(T_{\alpha, k}^{v} f\right)(z)=\sum_{i=1}^{d} \overline{v_{\alpha}\left(\alpha_{i}\right)} f_{\mid\left[\alpha_{i}, k\right]}(z) .
$$

When $\Gamma=\Gamma_{0}(N)$ (or any congruence subgroup of level $N$ ), one usually constructs Hecke operators $T_{n, k}^{v}$ corresponding to positive integers $n$ in which case $\alpha=\left(\begin{array}{ll}1 & 0 \\ 0 & n\end{array}\right)$ (cf. e.g. [3], [18, ch. 5], [55, ch. 9], 44] or [59] for more details).

4.5.1. Hecke operators for the theta multiplier system. Consider the case $\Gamma=\Gamma_{0}(4)$, $k=\frac{1}{2}$ and $v=v_{\theta}$. Let $n$ be a positive integer and $\alpha=\left(\begin{array}{ll}1 & 0 \\ 0 & n\end{array}\right)$. Then $g \in \overline{\Gamma_{0}(4)} \cap$ $\alpha^{-1} \overline{\Gamma_{0}(4)} \alpha$ can be written $g=\left(\begin{array}{cc}a & n b \\ c / n & d\end{array}\right)$, and $\alpha g \alpha^{-1}=\left(\begin{array}{ll}a & b \\ c & d\end{array}\right)$, with $a d-b c=1$ and $c \equiv 0 \bmod 4 n$. It is easy to verify that $v^{\alpha}(g)=v\left(\alpha g \alpha^{-1}\right)$ and $v(g)=$ $v\left(\alpha g \alpha^{-1}\right)\left(\frac{n}{d}\right)^{-1}$, and hence $v^{\alpha}(g)=v(g)$ if and only if $\left(\frac{n}{d}\right)=1$. By (4.8) this relation must hold for all odd integers $d$, and hence it is clear that the extension $v_{\alpha}$ exists if and only if $n$ is a perfect square. It is also easy to verify that in this case the multiplier system is given by $v_{\alpha}\left(g_{1} \alpha g_{2}\right)=v\left(g_{1}\right) v\left(g_{2}\right)$.

Suppose now for simplicity that $n=p^{2}$, with $p \neq 2$ a prime number. The $p^{2}+p$ different coset representatives of $\bar{\Gamma}$ in $\bar{\Gamma} \alpha \bar{\Gamma}$ are given by $\alpha_{b}=\left(\begin{array}{cc}1 & b \\ 0 & p^{2}\end{array}\right), b=$ $0, \ldots, p^{2}-1, \sigma=\left(\begin{array}{cc}p^{2} & 0 \\ 0 & 1\end{array}\right)$, and $\beta_{b}=\left(\begin{array}{cc}p & b \\ 0 & p\end{array}\right), b=1, \ldots, p-1$. By factorization of these representatives we see that $v_{\alpha}(\sigma)=v_{\alpha}\left(\alpha_{b}\right)=1, b=0, \ldots, p^{2}-1$ and $v_{\alpha}\left(\beta_{b}\right)=\epsilon_{p}\left(\frac{b}{p}\right)$ for $b=1, \ldots, p-1$. Hence, for $p \neq 2$

$$
\begin{aligned}
T_{p^{2}, \frac{1}{2}}^{v_{\theta}} f(z) & =\frac{1}{p}\left\{\sum_{b=0}^{p^{2}-1} \overline{v_{\alpha}}\left(\alpha_{b}\right) f\left(\alpha_{b} z\right)+\overline{v_{\alpha}}(\sigma) f(\sigma z)+\sum_{b=1}^{p-1} \overline{v_{\alpha}}\left(\beta_{b}\right) f\left(\beta_{b} z\right)\right\} \\
& =\frac{1}{p}\left\{\sum_{b=0}^{p^{2}-1} f\left(\frac{z+b}{p^{2}}\right)+f\left(p^{2} z\right)+\overline{\epsilon_{p}} \sum_{b=0}^{p-1}\left(\frac{b}{p}\right) f\left(z+\frac{b}{p}\right)\right\} .
\end{aligned}
$$

For $p=2$ the 4 coset representatives are given by $\alpha_{b}=\left(\begin{array}{ll}1 & b \\ 0 & 4\end{array}\right), b=0, \ldots, 3$, and we precisely obtain the operator in (4.2). The above construction is analogous to [60, pp. 450-451, thm. 1.7]. Suppose that $f \in \mathcal{M}\left(\Gamma_{0}(4), v_{\theta}, \frac{1}{2}, \lambda\right)$ has the following Fourier series at infinity:

$$
f(z)=\sum_{n \neq 0} \frac{a(n)}{\sqrt{|n|}} W_{\frac{1}{4} \operatorname{sgn}(n), i R}(4 \pi|n| y) e(n x)
$$

and that $T_{p^{2}, \frac{1}{2}}^{v_{\theta}} f(z)$ has a similar Fourier expansion but with coefficients $b^{(p)}(n)$. Using the formula for the standard Gauss sum, $\sum_{b=0}^{p^{2}-1} e\left(\frac{n b}{p^{2}}\right)=p^{2}$ if $p^{2} \mid n$ else 0 , 
and the twisted version $\sum_{b=1}^{p-1}\left(\frac{b}{p}\right) e\left(\frac{n b}{p}\right)=\sqrt{p}\left(\frac{n}{p}\right) \epsilon_{p}$ (cf. [7, Satz 7, p. 375] or [39. pp. 83-87]), we get that for all non-zero integers $n$

$$
\begin{aligned}
& b^{(p)}(n)=\left\{a\left(p^{2} n\right)+a\left(\frac{n}{p^{2}}\right)+p^{-\frac{1}{2}}\left(\frac{n}{p}\right) a(n)\right\}, p \neq 2, \text { and } \\
& b^{(2)}(n)=a(4 n) .
\end{aligned}
$$

(We use the standard convention that $a(x)=0$ if $x \notin \mathbb{Z}$.) It is now obvious that our Hecke operator $T_{p^{2}, \frac{1}{2}}^{v_{\theta}}$ is equal to the corresponding Hecke operator defined in [29, p. 199]. Observe that our Fourier coefficients $a(n)=\sqrt{n} \times$ Katok-Sarnak's Fourier coefficients $b(n)$.

As usual, we consider Hecke eigenforms in $\mathcal{M}\left(\Gamma_{0}(4), v_{\theta}, \frac{1}{2}, \lambda\right)$ which are simultaneous eigenfunctions of all $T_{p^{2}, \frac{1}{2}}^{v_{\theta}}$ with $p \neq 2$. An additional commuting normal operator can be chosen as either $T_{4, \frac{1}{2}}^{v_{\theta}}$ or $L$ (these two operators does not commute in general).

As it turns out, the operator $L$ is particularly useful in connection with the Shimura correspondence on the Kohnen space, $V^{+}$, where $L$ has eigenvalue 1 (cf. Section 6).

Observe that the Hecke eigenvalues in this case do not equal to the Fourier coefficients. In fact, suppose that $f$ as in (4.10) is an eigenfunction of all Hecke operators with $T_{p^{2}, \frac{1}{2}}^{v_{\theta}} f=\lambda_{p} f$ and that $a(t) \neq 0$ for some integer $t$. It is then easy to see that

$$
\begin{aligned}
& \lambda_{p}=\left\{\frac{a\left(t p^{2}\right)}{a(t)}+\frac{\left(\frac{t}{p}\right)}{\sqrt{p}}\right\}, p \neq 2, \text { and } \\
& \lambda_{2}=\frac{a(4 t)}{a(t)} .
\end{aligned}
$$

Using multiplicative relations of the Hecke operators one can prove (cf. [60, p. 453]) that if $t$ is square free, then

$$
a\left(t m^{2}\right) a\left(t n^{2}\right)=a(t) a\left(t m^{2} n^{2}\right), \text { for }(m, n)=1 .
$$

Furthermore, if $f$ is also an eigenfunction of $T_{4, \frac{1}{2}}^{v_{\theta}}$, then

$$
a(m) a(4 n)=a(4 m) a(n), m, n \in \mathbb{Z} .
$$

4.5.2. Hecke operators at integer weights and Fourier coefficients. Consider the modular group $\Gamma=P S L(2, \mathbb{Z})$, together with the integer weight $k \neq \equiv 0 \bmod 12$ and multiplier $v=v_{\eta}^{2 k}$.

It can be shown that for all positive integers $n$ and $m$ with $k n \equiv-k m \equiv k$ mod 12 we can construct Hecke operators $T_{n, k}^{v}$ and Hecke-like operators $\Theta_{m, k}^{v}$ acting on $\mathcal{M}(\Gamma, v, k, \lambda)$. Using these operators one can obtain multiplicative relations for Fourier coefficients similar to the weight zero case. This is shown in detail in [66], and here we will only state the theorem and give a brief outline of the ideas of the proof.

Theorem 1. Let $k$ be an integer $k \not \equiv 0 \bmod 12$ and $R>0$. Then there exists a basis of $\mathcal{M}(\Gamma, v, k, R)$ consisting of Maass wave forms $f$ with Fourier expansions at 
infinity,

$$
f(z)=\sum_{n=-\infty}^{\infty} \frac{c(n)}{\sqrt{\left|n+\frac{k}{12}\right|}} W_{\frac{k}{2} \operatorname{sgn}\left(n+\frac{k}{12}\right), i R}\left(4 \pi\left|n+\frac{k}{12}\right| y\right) e\left(\left(n+\frac{k}{12}\right) x\right),
$$

where the coefficients $c(n)$ satisfy the following multiplicative relations if $c(0) \neq 0$. For positive integers $m, n$ with $12 m \equiv 12 n \equiv 0 \bmod k$ set $m_{1}=\frac{12 m}{k}, n_{1}=\frac{12 n}{k}$ and $D=\frac{k}{(12, k)}$. If $\left(m_{1}+1, D\right)=\left(n_{1}+1, D\right)=1$

$$
c(m) c(n)=c(0) \sum_{0<d \mid\left(m_{1}+1, n_{1}+1\right)} \chi_{k}(d) c\left(\frac{k}{12}\left(\frac{\left(m_{1}+1\right)\left(n_{1}+1\right)}{d^{2}}-1\right)\right),
$$

and if $\left(m_{1}-1, D\right)=\left(n_{1}-1, D\right)=1$, then

$$
c(-m) c(-n)=\Lambda_{k, R} c(0) \sum_{0<d \mid\left(m_{1}-1, n_{1}-1\right)} \chi_{k}(d) c\left(\frac{k}{12}\left(\frac{\left(m_{1}-1\right)\left(n_{1}-1\right)}{d^{2}}-1\right)\right),
$$

where $\chi_{k}(d)=i^{k(d-1)}\left(=\left(\frac{-1}{d}\right)^{k}\right.$ for odd $\left.d\right)$ and

$$
\Lambda_{k, R}= \begin{cases}\prod_{j=1}^{l}\left(j(j-1)+\frac{1}{4}+R^{2}\right)^{2}, & k=2 l, \\ -R^{2} \prod_{j=1}^{l}\left(j^{2}+R^{2}\right)^{2}, & k=2 l+1 .\end{cases}
$$

In particular, we see that if $k \mid 12$ we have $D=1$, and the multiplicative relations (4.12) and (4.13) are valid for all positive integers.

Remark 8. As in the weight zero case and the coefficient $c(1)$ one can show that if $D=1$ and $f$ is an eigenfunction of all Hecke operators (defined below), then $c(0) \neq 0$ unless $f(z)$ is identically 0 . In the case $D>0$, if $c(0)=0$ and $f(z) \not \equiv 0$ we can choose an integer $n_{0}$ such that $c\left(n_{0}\right) \neq 0$ and obtain a similar set of multiplicative relations.

The proof of the "positive part" of the theorem, i.e. (4.12), relies on the construction of a family of Hecke operators $T_{m, k}^{v}$ with $k m \equiv k \bmod 12$. It is shown that this family consists of self-adjoint operators commuting with each other and the weight $k$ Laplacian. An explicit form of $T_{m, k}^{v}$ is

$$
T_{m, k}^{v} f(z)=\frac{1}{\sqrt{m}} \sum_{a d=m, d>0} \chi_{k}(d) \sum_{b=0}^{d-1} \overline{v(T)^{b d}} f\left(\frac{a z+b}{d}\right) .
$$

It is not hard to show that if $f(z)$ has Fourier coefficients $c(n)$, then $T_{m, k}^{v}$ has coefficients

$$
b(n)=\sum_{0<d \mid\left(m, n-\frac{k(m-1)}{12}\right)} \chi_{k}(d) c\left(\frac{n m}{d^{2}}+\frac{k\left(m-d^{2}\right)}{12 d^{2}}\right)
$$

from which we see that if $T_{m, k}^{v} f=\lambda_{m} f$ and $c(0) \neq 0$, then

$$
\lambda_{m}=\frac{1}{c(0)}\left[c\left(\frac{k(m-1)}{12}\right)+\chi_{k}(D) c\left(\frac{k\left(m-D^{2}\right)}{12 D^{2}}\right)\right] .
$$


The proof of (4.12) is concluded with a proof of the following multiplication rule (using straightforward calculations and induction). If $k m \equiv k n \equiv k \bmod 12$, then

$$
T_{m, k}^{v} T_{n, k}^{v}=\sum_{0<d \mid(m, n)} \chi_{k}(d) T_{\frac{m n}{d^{2}}, k}^{v} .
$$

To obtain the "negative part" of the theorem, i.e. (4.13), we have to consider another family of operators, $\Theta_{m, k}^{v}$ with $k m \equiv-k \bmod 12$. These operators are given as a combination of a bijection $\Theta=J \circ \mathcal{E}_{k}^{-}$mapping $\mathcal{N}(\Gamma, v, k, \lambda)$ to $\mathcal{M}(\Gamma, \bar{v}, k, \lambda)$ and a Hecke operator $T_{m, k}^{\bar{v}}$ mapping $\mathcal{M}(\Gamma, \bar{v}, k, \lambda)$ back to $\mathcal{M}(\Gamma, v, k, \lambda)$. Here $\mathcal{E}_{k}^{-}=$ $E_{2-k}^{-} \circ \cdots \circ E_{k-2}^{-} \circ E_{k}^{-}$maps $\mathcal{M}(\Gamma, v, k, \lambda)$ to $\mathcal{M}(\Gamma, v,-k, \lambda)$ bijectively, since $\lambda>\frac{1}{4}$ and $J$ reflects this space back to $\mathcal{M}(\Gamma, \bar{v}, k, \lambda)$. $T_{m, k}^{\bar{v}}$ is given by (4.15) with $v$ interchanged with $\bar{v}$. The operator $\Theta$ is similar to the operator defined by Maass in [42, p. 181]. Using (4.4)-(4.7) it is easy to show that $\Theta^{2} f=\Lambda_{k, R} f$ for all $f \in \mathcal{M}(\Gamma, k, v, \lambda)$ and that if $f(z)$ has Fourier coefficients $c(n)$, then $\Theta_{m, k}^{v} f(z)$ has coefficients

$$
d(n)=\sum_{0<d \mid\left(m, n-\frac{k(m+1)}{12}\right)} \chi_{k}(d) c^{\prime}\left(\frac{n m}{d^{2}}+\frac{k\left(m+d^{2}\right)}{12 d^{2}}\right)
$$

where

$$
c^{\prime}(n)=c(-n) \begin{cases}1, & n \geq 1 \\ \Lambda_{k, R}, & n \leq 0\end{cases}
$$

If $\Theta_{m, k}^{v} f=\mu_{m} f$ and $c(0) \neq 0$, then

$$
\mu_{m}=\frac{1}{c(0)}\left[c\left(\frac{k(m+1)}{12}\right)+\chi_{k}(D) c\left(\frac{k\left(m+D^{2}\right)}{12 D^{2}}\right)\right] .
$$

The multiplication rule for the operators $\Theta_{m, k}^{v}$ is that for $k m \equiv k n \equiv k \bmod 12$ we have

$$
\Theta_{m, k}^{v} \Theta_{n, k}^{v}=\Lambda_{k, R} \sum_{0<d \mid(m, n)} \chi_{k}(d) \Theta_{\frac{m n}{d^{2}}, k}^{v} .
$$

This in combination with the expressions for $\mu_{m}$ and $\lambda_{m}$ concludes the proof of (4.13).

Example 1. Look at the specific case $k=1$ and a function $f \in \mathcal{M}(\Gamma, v, 1, \lambda)$. Then by by setting $m=1$ in (4.12) and using the normalization $c(1)=1$ we see that

$$
c(n)=\sum_{0<d \mid(12 n+1,13)} \chi_{1}(d) c\left(\frac{\left(13(12 n+1)-d^{2}\right)}{12 d^{2}}\right),
$$

and hence if $(12 n+1,13)=1$, then we get a striking proportionality relation:

$$
c(n)=c(0) c\left(\frac{13(12 n+1)-1}{12}\right)=c(0) c(13 n+1) .
$$

For $12 n+1=13 l$ we get

$$
c(n)=c(0)\left(c(13 n+1)+c\left(\frac{n-1}{13}\right)\right),
$$

and if $(l, 13)=1$ we can combine these two equations and obtain

$$
c(n)=c(0)(c(13 n+1)+c(0) c(n)),
$$


and hence

$$
c(n)=\frac{c(0)}{1-c(0)^{2}} c(13 n+1) .
$$

Now consider $m=-1$ in (4.13) and note that $\Lambda_{1, R}=-R^{2}$. If $(12 n-1,11)=1$, then

$$
c(-n)=\frac{-R^{2} c(0)}{c(-1)} c(11 n-1),
$$

and if $(12 n-1,11)=11$, then

$$
c(-n)=\frac{-R^{2} c(0)}{c(-1)}\left[c(11 n-1)-c\left(\frac{n-1}{11}\right)\right] .
$$

We have not seen relations of type (4.16)-(4.21) earlier in the literature. For some numerical examples see Tables 2 and 3. Examples of relations for higher weights can be found in [66].

Remark 9. An alternative approach to the above coefficient relations in the case of weight 1 is to identify $\mathcal{M}\left(\Gamma_{0}(1), v_{\eta}^{2}, 1, \lambda\right)$ with a subspace of $\mathcal{M}\left(\Gamma_{0}(144),\left(\frac{-1}{d}\right), 1, \lambda\right)$ via the map $f(z) \mapsto g(z)=f(12 z)$. Cf. e.g. [64, sec. 2.4.7].

This identification also provides an explanation for the occurrence of CM-type forms found (numerically) in $\mathcal{M}\left(\Gamma_{0}(1), v_{\eta}^{2}, 1, \lambda\right)$. These forms have eigenvalues in an arithmetic progression: $R_{k}=\frac{2 \pi k}{\ln (7+2 \sqrt{12})}$ for $k \in \mathbb{Z}$. See Table 4

\section{The Eisenstein Series for $P S L(2, \mathbb{Z})$ AT Weight Zero}

In case there is a cusp $p_{j}$ at which the multiplier system is singular (i.e. $v\left(S_{j}\right)=$ 1) we have a continuous spectrum, $\left[\frac{1}{4}, \infty\right.$ ) (with multiplicity equal to the number of singular cusps), and in general we cannot say much about the embedded discrete spectrum in $\left[\frac{1}{4}, \infty\right)$.

Examples of singular cusps are the cusp at infinity for the eta multiplier and weight $k \equiv 0 \bmod 12$ on $\operatorname{PSL}(2, \mathbb{Z})$ and the cusps at 0 and $i \infty$ for the theta multiplier and weight $\frac{1}{2}$ on $\Gamma_{0}(4)$.

Remember that Maass waveforms are part of the discrete spectrum, but as we continuously "turn off" the multiplier system, i.e. for $v=v_{\eta}^{2 k}$ we let $k \rightarrow 0$, the continuous spectrum will emerge in the limit. For this reason we want to review some details concerning the Eisenstein series on the modular group.

At weight 0 and singular character $\chi$, the continuous spectrum of $\Delta=\Delta_{0}$ is the interval $\left[\frac{1}{4}, \infty\right)$, and the eigenpacket is given by the Eisenstein series $E(z ; s ; \chi)$ defined by

$$
E(z ; s ; \chi)=\sum_{T \in \Gamma_{\infty} \backslash \Gamma} \chi\left(T^{-1}\right)(\Im(T z))^{s}
$$

where $\Gamma_{\infty}=[S]$. For the trivial character we have the Fourier series expansion (cf. [22, p. 65 and p. 76])

$$
E(z ; s ; \chi)=y^{s}+\varphi(s) y^{1-s} \sum_{n \neq 0} \varphi_{n}(s) \sqrt{y} K_{s-\frac{1}{2}}(2 \pi|n| y) e(n x),
$$


where

$$
\begin{aligned}
\varphi(s) & =\sqrt{\pi} \frac{\Gamma\left(s-\frac{1}{2}\right)}{\Gamma(s)} \frac{\zeta(2 s-1)}{\zeta(2 s)}, \text { and } \\
\varphi_{n}(s) & =\frac{2 \pi^{s}|n|^{s-\frac{1}{2}}}{\Gamma(s)} \frac{\sigma_{1-2 s}(|n|)}{\zeta(2 s)} .
\end{aligned}
$$

Hence we can see that for $s=\frac{1}{2}+i R$, the $n$th Fourier coefficient of $E(z ; s)$ is given by

$$
\begin{aligned}
c(n) & =\varphi_{n}\left(\frac{1}{2}+i R\right)=K \cdot|n|^{i R} \sigma_{-2 i R}(|n|) \\
& =K \cdot|n|^{i R} \sum_{d|| n \mid, d>0} d^{-2 i R},
\end{aligned}
$$

where $K=K(R)$ is a constant dependent on $R$. For a prime $p$ we get

$$
c(p)=K \cdot p^{i R}\left(1+p^{-2 i R}\right)=2 K \cdot \cos (R \ln p) .
$$

Using this formula we can compute quotients of various $c(p)$ (e.g. $\left.\frac{c(2)}{c(3)}\right)$ and compare this with corresponding quotients for the experimentally obtained forms of weight $k \approx 0$.

\section{The Shimura Correspondence}

We know that the $\theta$-function is an automorphic form (not a cusp form) of weight $\frac{1}{2}$ on $\Gamma_{0}(4)$; hence we consider $\Gamma_{0}(4)$ together with the $\theta$-multiplier system (cf. Section [2.3).

6.1. Introduction - the holomorphic case. We will consider the Shimura correspondence only in the particular case of trivial character and will level a squarefree multiple of 4 . Let $S_{k}(N)$ denote the space of holomorphic cusp forms of weight $k \in \mathbb{Z}$ (and trivial multiplier) on $\Gamma_{0}(N)$, and let $S_{k+\frac{1}{2}}(4 N)$ denote the space of holomorphic cusp forms of weight $k+\frac{1}{2}, k \in \mathbb{Z}$, and multiplier $v_{\theta}$ on $\Gamma_{0}(4 N)$. The Shimura correspondence is a correspondence between the space $S_{k+\frac{1}{2}}(4 N)$ and spaces $S_{2 k}\left(N^{\prime}\right)$ for certain integers $N^{\prime} \mid 4 N$ (e.g. $N^{\prime}=2 N$ or $N$ ).

The map from $S_{k+\frac{1}{2}}$ to $S_{2 k}$ was first constructed by Shimura [60], and later an adjoint map from $S_{2 k}$ to $S_{k+\frac{1}{2}}$ was constructed by Shintani [62. The former uses a Dirichlet series and the latter uses an integral against a theta function. Both of these maps commute with the Hecke operators that are acting on $S_{2 k}(N)$ and $S_{k+\frac{1}{2}}(4 N)$ respectively. Kohnen [32, 33] proved that for $N$ odd and square-free, the correspondence is a bijection between the newforms on $S_{2 k}(N)$ and a certain subspace, $V^{+} \subseteq S_{k+\frac{1}{2}}(4 N)$. The subspace $V^{+}$is composed of Hecke eigenfunctions whose Fourier coefficients, $c(n)$, satisfy certain vanishing properties; namely, $c(n)=$ 0 for $n \equiv 2,3 \bmod 4$ (see also Section 4.3).

Following from the Shimura correspondence is a connection between certain Fourier coefficients of the half integral weight forms and critical values of the twisted L-series for the corresponding integral weight form. Cf. e.g. 73, 74, 35, 34] and 61. 
6.2. The Shimura correspondence for Maass waveforms. The extension of the Shimura correspondence and Kohnen's result to spaces of Maass waveforms has been investigated by e.g. Sarnak [57], Hejhal [20], Duke [14, Katok-Sarnak [29], Khuri-Makdisi [30, Kojima [36, 37, 38, Biró [5] and Arakawa [2. Of these, 30] and [37, 38, are written in the more general setting of Hilbert modular forms for number fields. Reading [30] together with [61] and 29] gives a good picture of the current state of affairs.

Throughout this section let $\mathcal{M}(N, R)^{+}$denote the space of even (with respect to $J: z \mapsto-\bar{z})$ Hecke normalized Maass waveforms in $\mathcal{M}\left(\Gamma_{0}(N), 1,0, R\right)$ and let $\mathcal{M}_{\frac{1}{2}}(4, R)$ denote the space of Hecke normalized (with respect to all $T_{p^{2}}, p \neq 2$ ) weight $\frac{1}{2}$ Maass waveforms in $\mathcal{M}\left(\Gamma_{0}(4), v_{\theta}, \frac{1}{2}, R\right)$. Also let $V^{+} \subseteq \mathcal{M}_{\frac{1}{2}}(4, R)$ be defined as in Section 4.3 .

For $f \in \mathcal{M}(N, R)^{+}$and $\phi \in \mathcal{M}_{\frac{1}{2}}(4, R)$ we will use the following notation:

$$
f(z)=\sum_{\substack{n=-\infty \\ n \neq 0}}^{\infty} A(n) \sqrt{y} K_{i R}(2 \pi|n| y) e(n x)
$$

and

$$
\phi(z)=\sum_{\substack{n=-\infty \\ n \neq 0}}^{\infty} \frac{a(n)}{\sqrt{|n|}} W_{\frac{1}{4} \operatorname{sgn}(n), i R}(4 \pi|n| y) e(n x)
$$

(Both expansions are given with respect to the cusp at $\infty$.)

The existence of a Shimura correspondence and an inverse for Maass waveforms is expressed by the following proposition (essentially [30, thm. 5.1 and 5.2]):

\section{Proposition 5.}

a) The Shimura correspondence gives a map $\Phi: \mathcal{M}_{\frac{1}{2}}(4, R) \rightarrow \mathcal{M}(2,2 R)^{+}$.

b) Conversely, let $f \in \mathcal{M}(2,2 R)^{+}$. Then there exists a $\phi \in \Phi^{-1}(f) \in \mathcal{M}_{\frac{1}{2}}(4, R)$.

c) If $A(p)$ is the Hecke eigenvalue of $f$ with respect to the operator $T_{p}^{2}$ and $\lambda_{p}$ is the eigenvalue of $\phi \in \Phi^{-1}(f)$ with respect to $T_{p^{2}, \frac{1}{2}}^{v_{\theta}}$, then we actually have

$$
A(p)=\lambda_{p}
$$

Proof. See the proof of [30, thm. 5.1 and 5.2]. For c) observe the difference in normalization of the Hecke operators. Cf. also [36, thm. 2], Proposition 6] a) and (4.11).

Remark 10. From [29, props. 4.1 and 2.3] we know that the above correspondence $\Phi$ actually restricts to a map between $\mathcal{M}(1,2 R)^{+}$and the subspace $V^{+}$. Hence a new form in $\mathcal{M}(2,2 R)^{+}$will be mapped to $V^{-}$, the orthogonal complement of $V^{+}$. Vice versa, a form in $V^{-}$will be mapped to a new form in $\mathcal{M}(2,2 R)^{+}$.

To generalize the results mentioned at the end of the previous subsection to Maass waveforms we need the following definition. For $f \in \mathcal{M}(2, R)^{+}$with Fourier coefficients $\{A(n)\}$ and a given Dirichlet character $\chi$, we define the $\chi$-twisted Lseries of $f$ by

$$
L(f, \chi, s)=\sum_{n \neq 0}^{\infty} A(n) \chi(n)|n|^{-s-\frac{1}{2}}
$$


Proposition 6. Let $\phi \in \mathcal{M}_{\frac{1}{2}}(4, R)$ have Fourier coefficients $\{a(n)\}$ and correspond (via Proposition 5) to $f=\Phi(\phi) \in \mathcal{M}(N, 2 R)^{+}$(where $N=1$ if and only if $\phi \in V^{+}$, otherwise $N=2$ ) with Fourier coefficients $\{A(n)\}$. Let $t \in \mathbb{Z}^{+}$be square-free and let $\chi_{t}^{\prime}$ be the quadratic residue symbol $\left(\frac{t}{)}\right)$ considered $\bmod N t$ (i.e. we have $\chi_{t}^{\prime}(n)=\left(\frac{t}{n}\right)$ if $(n, N)=1$ and $\chi_{t}^{\prime}(n)=0$ otherwise).

Then the following properties hold:

a) If $a(t) \neq 0$, then $A(n)$ can be expressed by

$$
A(n)=\sum_{\substack{k d=n \\ k>0}} \frac{\chi_{t}^{\prime}(k)}{\sqrt{k}} \frac{a\left(t d^{2}\right)}{a(t)}, n \in \mathbb{Z}^{+} .
$$

To express a $(t)$ in terms of $A(n)$ 's we get four cases:

b) If $f$ is an oldform, i.e. $f \in \mathcal{M}(1,2 R)^{+}$, then $\phi \in V^{+}$, and hence

$$
a(t)=0, t \equiv 2,3 \bmod 4 \text {. }
$$

c) If $f$ is a newform with eigenvalue $\epsilon$ with respect to the involution $z \mapsto \frac{-1}{2 z}$, then

$$
a(t)=0
$$

for $t \equiv 5 \bmod 8$ if $\epsilon=1$ and for $t \equiv 1 \bmod 8$ if $\epsilon=-1$.

d) For all other (square-free) values of the following formula holds:

$$
|a(t)|^{2}=Q \frac{\langle\phi, \phi\rangle}{\langle f, f\rangle} L\left(f, \chi_{t}, 0\right),
$$

where $Q$ is a constant independent of $t$.

e) If $\phi$ is a normalized Hecke eigenform (for all $T_{p^{2}, \frac{1}{2}}^{v_{\theta}}, p$ an odd prime), then $f$ is also a normalized Hecke eigenform.

Proof. Cf. 60, pp. 448 (prop. 1.3), 458 (main theorem), 474 (line -11)], and [30, p. 422] for the choice of $\chi_{t}^{\prime}$ (the $N=1$ case is simply to incorporate the results of [29]). Relation a) follows from [30, thm. 5.1 (2)], which in our case can be written as

$$
\sum_{n=1}^{\infty} A(n) n^{-s}=c \cdot L\left(s+\frac{1}{2}, \chi_{t}^{\prime}\right) \sum_{n=1}^{\infty} a\left(t n^{2}\right) n^{-s} .
$$

(Cf. e.g. also [60, p. 458], [47, p. 159], 61, prop. 3.1].) The relation b) follows from [29. prop. 2.3] (see also [32]), and relation c) is implicit in [30, thms. 8.1 (8.6)]. Simply observe that in our normalization the relevant factor in the product is given by

$$
\left(\sqrt{2} A(2)-\left(\frac{2}{t}\right)\right)=-\left(\epsilon+\left(\frac{2}{t}\right)\right)
$$

since $A(2)=\frac{-\epsilon}{\sqrt{2}}$ for a newform (analogous to [3, thm. 3]). (Alternatively consider the sign of the functional equation for $L\left(f, \chi_{t}, s\right)$.) Relation d) is the Maass waveform-analogue of [35, thm. 1] and follows from [30, thm. 8.1] (in the notation of [30] we have $\left.a(n)=|n|^{\frac{1}{4}} \mu(n ; \phi)\right)$.

Remark 11. That the map $\Phi$ is well-defined and surjective from $\mathcal{M}_{\frac{1}{2}}(4, R)$ onto $\mathcal{M}(2,2 R)^{+}$follows from [30, thm. 5.1 and 5.2] and "multiplicity one" for the Hecke operators $T_{p}$ on $\mathcal{M}(2,2 R)^{+}$(cf. 68, thm. 4.6]). Experimentally we have observed that $\Phi$ restricted to $V^{+}$also seems to be injective. Theoretically, this is still an 
open problem that might be possible to resolve using the trace formula for the Hecke operators $T_{p^{2}}$ on $V^{+}$.

Remark 12. Note that the same argument as for Proposition 6 c) implies that to any $f \in \mathcal{M}(2,2 R)^{+}$with Fourier coefficient $A(2)=\frac{ \pm 1}{\sqrt{2}}$ there corresponds a function $\phi \in \Phi^{-1}(f) \subseteq \mathcal{M}_{\frac{1}{2}}(4, R)$ with coefficients $a(n)=0$ for either $n \equiv 1$ or 5 mod 8 respectively. Hence, since oldforms on $\Gamma_{0}(2)$ occur in pairs, we can choose two forms $f_{1}, f_{2}$ with $A_{1}(2)=\frac{1}{\sqrt{2}}$ respectively $A_{2}(2)=\frac{-1}{\sqrt{2}}$. These two functions thus correspond to two linearly independent non-zero functions in $\mathcal{M}_{\frac{1}{2}}(4, R)$, which hence is at least two dimensional when $2 R$ is an even eigenvalue for $\operatorname{PSL}(2, \mathbb{Z})$.

The relations a)-c) in Proposition [6 appeared in [64, ch. 2] as experimental observations (cf. 8.4, in particular Tables 5.88).

See also [12, §4.1] and [13, p. 633], and [61, pp. 502 (bottom) - 504 (top)] for some additional perspectives.

\section{Some computational Remarks}

We recall the key ingredients in the standard Hejhal's algorithm (cf. e.g. 25, 26, 27, 65, 64 and 6]) to compute weight zero Maass waveforms on cofinite Fuchsian groups. First of all the asymptotic properties of the K-Bessel function are used to obtain an approximation of the Maass waveform by a truncated Fourier series. By viewing this as a discrete Fourier transform one can use the inverse transform to express any coefficient as a linear combination of the other coefficients. Finally, by using the assumed automorphy properties of the function one obtains a non-trivial linear system that is satisfied by the Fourier coefficients.

To use this algorithm to also locate eigenvalues the most general method is to use two different sets of sampling points for the inverse transform and try to minimize the difference between the correspondingly computed solution vectors.

The following two modifications are needed in order to make the weight zero algorithm work in the general case:

- the K-Bessel function needs to be replaced with the Whittaker function, and

- the automorphy condition needs to incorporate the multiplier system.

The first modification, although trivial in theory, is the most complex in terms of the numerics involved. There was no efficient algorithm for the Whittaker function in the literature, and a new algorithm had to be developed. We used the integral representation (cf. [22, p. 375 (top)])

$$
W_{k, i R}(2 x)=\sqrt{\frac{2 x}{\pi}} \int_{0}^{\infty} e^{-x \cosh t} \Psi\left[-k ; \frac{1}{2} ; x(1+\cosh t)\right] \cosh [i R t] d t,
$$

where $\Psi$ is a confluent hypergeometric function together with a stationary phase method, to develop a robust and efficient algorithm. This algorithm is in essence similar to Hejhal's algorithm (cf. [25) for the K-Bessel function, $K_{i R}(x)$, and the generalization of it made by Avelin (cf. [4, Avelin]) to a complex argument, $K_{s}(x)$, $s \in \mathbb{C}$. The details of this algorithm are described in [64, Ch. 4].

Let $\Gamma$ be a Fuchsian group with fundamental domain $\mathcal{F}$ and let $p_{j}, \sigma_{j}, 1 \leq j \leq \kappa$, $q_{i}$ and $U_{i}, 1 \leq i \leq \kappa_{0}$, be as in Section 1.2 Let $I(w)=i$ if $q_{i}$ is the closest (in the hyperbolic metric) parabolic vertex to $w \in \mathcal{F}$. 
Consider $z \in \mathcal{H}$ and let $T_{j} \in \Gamma$ be the pull-back map of $\sigma_{j} z$, i.e. $T_{j} \sigma_{j} z \in \mathcal{F}$. Observe that $f_{j}(z)=f_{\mid \sigma_{j}}(z)=j_{\sigma_{j}}(z ; k)^{-1} f\left(\sigma_{j} z\right)$, and with $z_{j}^{*}=\sigma_{I(j)}^{-1} U_{I(j)} T_{j} \sigma_{j} z$, where we set $I(j)=I\left(T_{j} \sigma_{j} z\right)$ (cf. 65, p. 23]), the automorphy condition now becomes

$$
\begin{aligned}
f_{j}(z) & =j_{\sigma_{j}}(z ; k)^{-1} f\left(\sigma_{j} z\right)=j_{\sigma_{j}}(z ; k)^{-1} f\left(T_{j}^{-1} U_{I(j)}^{-1} \sigma_{I(j)} z_{j}^{*}\right) \\
& =j_{\sigma_{j}}(z ; k)^{-1} v\left(T_{j}^{-1} U_{I(j)}^{-1}, k\right) j_{T_{j}^{-1} U_{I(j)}^{-1}}\left(\sigma_{I(j)} z_{j}^{*} ; k\right) f\left(\sigma_{I(j)} z_{j}^{*}\right) \\
& =j_{\sigma_{j}}(z ; k)^{-1} j_{T_{j}^{-1} U_{I(j)}^{-1}}\left(\sigma_{I(j)} z_{j}^{*} ; k\right) j_{\sigma_{I(j)}}\left(z_{j}^{*} ; k\right) v\left(T_{j}^{-1} U_{I(j)}^{-1}, k\right) f_{I(j)}\left(z_{j}^{*}\right) .
\end{aligned}
$$

The entire setup of the Phase 1 algorithm, i.e. locating eigenvalues and computing the first few Fourier coefficients, goes through exactly as in the case of weight 0 (cf. 65] or [64, Ch. 1]) with some trivial modifications. For the sake of completeness we will give an outline of the modified algorithm. Consider $f \in \mathcal{M}(\Gamma, v, k, \lambda)$, and using the notation $n_{i}=n+\alpha_{i}, f$ has a Fourier series expansion at the cusp $p_{i}$ :

$$
f_{i}(z)=f_{\mid\left[k, \sigma_{j}\right]}(z)=\sum_{\substack{-\infty \\ n+\alpha_{i} \neq 0}}^{\infty} \frac{c_{i}(n)}{\sqrt{\left|n_{i}\right|}} W_{\frac{k}{2} \operatorname{sgn}\left(n_{i}\right), i R}\left(4 \pi\left|n_{i}\right| y\right) e\left(n_{i} x\right),
$$

and since the Whittaker function is ultimately exponentially decaying, given an $\epsilon>0$, there exists a constant (depending on $y$ and $\epsilon$ ), $M(y)$, such that

$$
f_{i}(z)=\hat{f}_{i}(z)+[[\epsilon]]
$$

where we use $[[\epsilon]]$ to denote a constant of magnitude less than $\epsilon$. The truncated Fourier series

$$
\hat{f}_{i}(z)=\sum_{\substack{-M(Y) \\ n+\alpha_{i} \neq 0}}^{M(Y)} \frac{c_{i}(n)}{\sqrt{\left|n_{i}\right|}} W_{\frac{k}{2} \operatorname{sgn}\left(n_{i}\right), i R}\left(4 \pi\left|n_{i}\right| y\right) e\left(n_{i} x\right)
$$

is now viewed as a discrete Fourier transform, and if we take the inverse transform over the horocyclic points $z_{m}=x_{m}+i Y, 1-Q \leq m \leq Q$, where $x_{m}=\frac{1}{2 Q}\left(\frac{1}{2}-m\right)$, we get

$$
\begin{aligned}
& \frac{1}{2 Q} \sum_{1-Q}^{Q} \hat{f}_{i}\left(z_{m}\right) e\left(-n_{i} x_{m}\right) \\
& =\frac{1}{2 Q} \sum_{m=1-Q}^{Q} \sum_{\substack{l=-M(Y) \\
l_{i} \neq 0}}^{M(Y)} \frac{c_{i}(l)}{\sqrt{\left|l_{i}\right|}} W_{\frac{k}{2} \operatorname{sgn}\left(l_{i}\right), i R}\left(4 \pi\left|l_{i}\right| Y\right) e\left(l_{i} x_{m}-n_{i} x_{m}\right) \\
& =\sum_{l=-M(Y)}^{M(Y)} \frac{c_{i}(l)}{\sqrt{\left|l_{i}\right|}} W_{\frac{k}{2} \operatorname{sgn}\left(l_{i}\right), i R}\left(4 \pi\left|l_{i}\right| Y\right) \frac{1}{2 Q} \sum_{1-Q}^{Q} e\left(l_{i} x_{m}-n_{i} x_{m}\right) \\
& =\frac{c_{i}(n)}{\sqrt{\left|n_{i}\right|}} W_{\frac{k}{2} \operatorname{sgn}\left(n_{i}\right), i R}\left(4 \pi\left|n_{i}\right| Y\right),
\end{aligned}
$$

where we used the fact that

$$
\frac{1}{2 Q} \sum_{1-Q}^{Q} e\left(l_{i} x_{m}-n_{i} x_{n}\right)=e\left(\frac{l_{i}-n_{i}}{4 Q}\right) \frac{1}{2 Q} \sum_{1-Q}^{Q} e\left(-\left(l_{i}-n_{i}\right) \frac{m}{2 Q}\right)=\delta_{n l} .
$$


Now we also have $f_{i}\left(z_{m}\right)=\chi_{m i} f_{I(m, i)}\left(z_{m i}^{*}\right)$, where (by (7.1))

$$
\chi_{m i}=j_{\sigma_{i}}\left(z_{m} ; k\right)^{-1} j_{T_{i}^{-1} U_{I(m, i)}^{-1} \sigma_{I(m, i)}}\left(z_{m i}^{*} ; k\right) w\left(T_{i}^{-1} U_{I(m, i)}^{-1}, \sigma_{I(m, i)}\right) v\left(T_{i}^{-1} U_{I(m, i)}^{-1}, k\right) .
$$

Hence

$$
\begin{aligned}
\frac{c_{i}(n)}{\sqrt{\left|n_{i}\right|} W_{s g n\left(n_{i}\right) \frac{k}{2}, i R}\left(4 \pi\left|n_{i}\right| y\right)} & =\frac{1}{2 Q} \sum_{1-Q}^{Q} f_{i}\left(z_{m}\right) e\left(-n_{i} x_{m}\right)+[[\epsilon]] \\
& =\frac{1}{2 Q} \sum_{1-Q}^{Q} \chi_{m i} f_{I(m, i)}\left(z_{m i}^{*}\right) e\left(-n_{i} x_{m}\right)+[[\epsilon]] \\
& =\sum_{j=1}^{\kappa} \sum_{\substack{l=-M_{0} \\
n+\alpha_{i} \neq 0}}^{M_{0}} c_{j}(l) V_{n l}^{i j}+2[[\epsilon]],
\end{aligned}
$$

where

$$
V_{n l}^{i j}=\frac{1}{\sqrt{\left|l_{j}\right|}} \frac{1}{2 Q} \sum_{\substack{1-Q \\ I(m, i)=j}}^{Q} \chi_{m i} W_{s g n\left(l_{j}\right) \frac{k}{2}, i R}\left(4 \pi\left|l_{j}\right| y_{m j}^{*}\right) \times e\left(l_{j} x_{m j}^{*}\right) e\left(-n_{i} x_{m}\right) .
$$

We then define $\tilde{V}_{n l}^{i j}$ by

$$
\tilde{V}_{n l}^{i j}=V_{n l}^{i j}-\delta_{n l} \delta_{j i} \frac{1}{\sqrt{\left|n_{i}\right|}} W_{s g n\left(n_{i}\right) \frac{k}{2}, i R}\left(4 \pi\left|n_{i}\right| Y\right)
$$

and if we neglect the error of magnitude $\epsilon$ we finally obtain a (well-conditioned) linear system

$$
C V=0,
$$

which must be satisfied by the Fourier coefficients of $f$. Here $V$ is the matrix $\tilde{V}_{n l}^{i j}$ and $C$ is the vector $c_{i}(n)$, both depending on $R$ and $Y$. The basic idea of Phase 1 is now to locate eigenvalues $R$ together with sets of corresponding Fourier coefficients, $c_{i}(n)$, by solving $\left(^{*}\right)$ repeatedly for different $R$ 's, and seeking those values of $R$ for which the solution vectors $C=C(R, Y)$ are stable under changes of $Y$. That is, if $C\left(R, Y_{1}\right) \approx C\left(R, Y_{2}\right)$ (for $Y_{1}$ and $Y_{2}<Y_{0}$, for some suitable constant $Y_{0}$ ), we take it as an indication that $R$ is close to an eigenvalue and that the components of $C$ are close to the corresponding Fourier coefficients. For more details and justifications see [65, sect. 3.2].

We note that the Phase 2 algorithm (i.e. computation of a larger set of Fourier coefficients) also generalizes to non-zero weight in a similar manner (cf. 65, sect. $3.3])$.

\section{NumericAl RESUlts}

The experimental excursions have been directed towards three essentially different subjects, but, in each, we have worked in an exploratory spirit:

- First, we tried to get an overall picture of the distribution of small to middle-range sized eigenvalues on $\operatorname{PSL}(2, \mathbb{Z})$ (and the eta multiplier) for "large" weights, e.g. $R \in[0,14]$ and $k \in[0.1,6]$. 
- Second, we continuously "turned off" the multiplier system $v_{\eta}^{2 k}$ on $\operatorname{PSL}(2, \mathbb{Z})$ by letting the weight $k \rightarrow 0$ and studied the varying distribution of eigenvalues, $N_{k}(T)$, as well as the formation of the continuous spectrum.

- There are some cases where arithmeticity plays a role even in non-zero weight. We studied the Shimura correspondence between weight zero forms on $\Gamma_{0}(2)$ and weight one-half forms on $\Gamma_{0}(4)$. We also studied weight one forms on $P S L(2, \mathbb{Z})$ which correspond to Hecke eigenforms on $\Gamma_{0}(144)$ with a Dirichlet character.

8.1. Varying weight. The first experiment considered was to tabulate the first few eigenvalues (up to $R=14$ ) for $\operatorname{PSL}(2, \mathbb{Z})$ and the multiplier system $v_{\eta}^{2 k}$ of weight $k \in(0,6]$ (cf. Section 4.4.1). We made the computations for $k \in[0.1,6]$ with a grid size of 0.01 , and the results are presented in Figure 1, We stress here that the arcs in Figure 1 terminate at $k=0.1$; it is not excluded (and actually expected) that they might go lower.

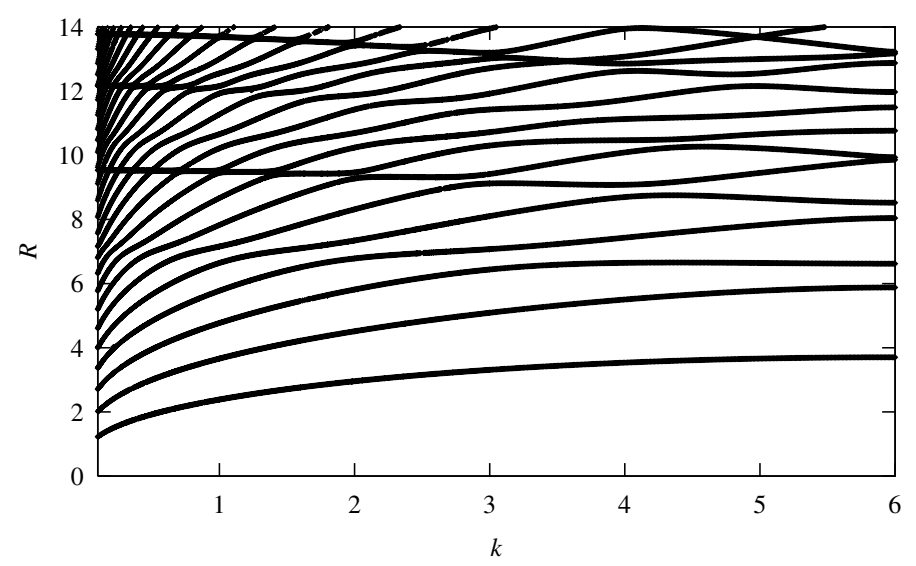

Figure 1. Plot of spectral parameters $R=R_{j}(k)$ with $(R, k) \in$ $[0,14] \times[0.1,6]($ cf. Section 8.1) .

For some examples of eigenvalues for "large" weights see Table 1. This data should be compared with data obtained by Mühlenbruch, [46, p. 160], who used a completely different method (with much less accuracy). We note here that as $R$ increases, the negative Fourier coefficients seem to grow rapidly in magnitude (as compared to the positive ones, with the normalization $c(1)=1$ ) for "large" weights.

We believe that we have found all eigenvalues with $(R, k) \in[0,14] \times[0.1,6]$. This belief is founded on the "continuity" of the resulting graphs $R_{j}(k)$ (cf. Figure 1), where $R_{j}(k)$ is the $j$ th eigenvalue at weight $k$, considered as a function of $k$. By standard results (e.g. [10, p. 149]) $R_{j}(k)$ should be a real analytic function in this interval.

Remember that, for $k \geq 0$, the smallest eigenvalue, $\lambda_{\min }$, corresponds to the function $F(z)=y^{\frac{k}{2}} \eta(z)^{2 k}$. A lower bound for the second smallest eigenvalue, $\lambda_{0}(k)$, is discussed in [10, p. 183]. Bruggeman finds two such bounds, both positive, which he calls $\mu_{0}(k)$ and $\mu_{1}(k)\left(\mu_{1}\right.$ is better than $\mu_{0}$ in a certain interval $\left.I \subset[0,2]\right)$. Figure 2 shows a comparison between the $R$-values corresponding to these bounds and the smallest experimentally found eigenvalues in the interval $k \in[0.1,6]$; we see that Bruggeman's bounds can be improved quite a bit. 


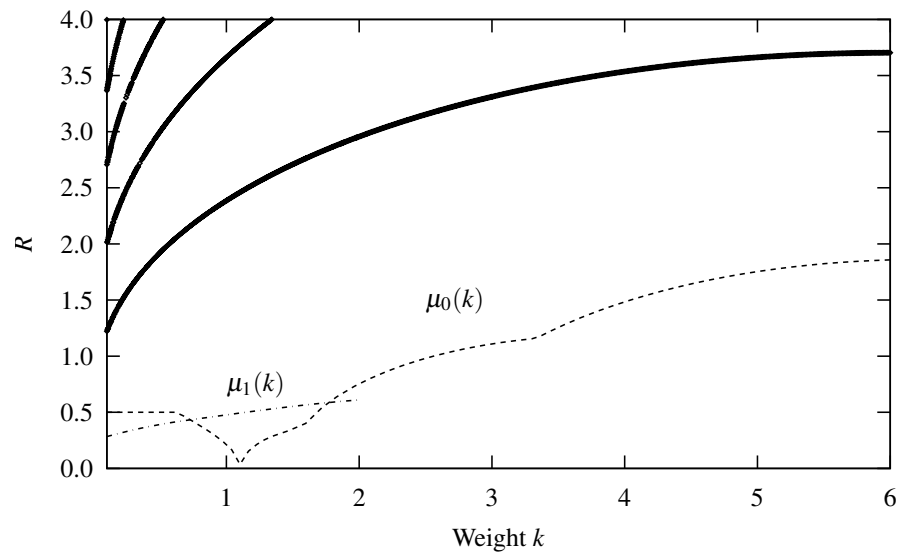

Figure 2. Comparison with the theoretical lower bounds in $k \in[0.1,6]$.

8.2. Small weights. The investigation of eigenvalues for small weights $k$ has been done in the interval $R \in[0,20]$, and $k \in\left\{10^{-j} \mid 1 \leq j \leq 12\right\} \subset\left[10^{-12}, 0.1\right]$. We believe that most cusp forms were found. Let us use the notation

$$
\lambda_{j}(k)=\frac{1}{4}+R_{j}^{2}(k)
$$

for the $j$ th discrete eigenvalue at weight $k$, and $\phi_{j}(k)$ for the corresponding cusp form. It is then a basic fact that $\lambda_{j}(k)$ depends continuously on $k$, but it can also be shown that for $k \in(0,12) \lambda_{j}$ is even real analytic in $k$. That is, $\phi_{j}(k)$ belongs to an "analytic family" in the terminology of Bruggeman (see [10, 11]). In connection with this, it should also be noted that our experiments support the statement in Observation 71 in [46] that the first few cusp forms at weight 0 do not belong to an analytic family of cusp forms defined in the interval $(-12,12)$. Indeed we find that in the range considered we actually seem to have $\lambda_{j}(k) \rightarrow 0$ as $k \rightarrow 0$, which is consistent with Bruggeman's result (cf. case ii) b) in Prop. 2.17 in [10, p. 149]).

Our experiments indicate that for fixed small $k$, the "generic" cusp forms $\phi_{j}(k)$ can be divided into two classes:

- $C(k)$ and

- $E(k)$.

Here $C(k)$ consists of functions $\phi_{j}(k)$ such that $\lambda_{j}(k)$ is close to an eigenvalue of a cusp form at weight 0 and the Fourier coefficients are close to the corresponding coefficients of the weight-zero cusp form.

$E(k)$ on the other hand consists of functions $\phi_{j}(k)$ such that the Fourier coefficients are close to the Fourier coefficients of the Eisenstein series $E(z, s)$ where $\lambda_{j}(k)=s(1-s)$.

The typical difference between the Fourier coefficients at weight $k$ and weight 0 are in both cases basically of order $k$; for the forms in $C(k)$, the distance between $\lambda_{j}(0)$ and the corresponding discrete eigenvalue at weight 0 is much smaller than $k$.

The "generic" in the statement above means that we exclude certain places where the families $\phi_{j}(k)$ change character between $C(k)$ and $E(k)$. In these cases we have 
a situation of almost multiplicity 2, and in too coarse resolution it actually looks like the analytic families intersect.

Weyl's law for a non-trivial $\eta$-multiplier and a fixed non-zero weight $k \in(0,12)$ on $\operatorname{PSL}(2, \mathbb{Z})$ is

$$
N_{k}(T)=\#\{R \leq T, \text { weight } k\}=\frac{T^{2}}{12}-\frac{T}{\pi} \ln \left|1-e^{\frac{k \pi}{6} i}\right|+S(T)+O(1),
$$

for $T \geq 1$ (cf. [22, p. 466] with $\kappa_{0}=0$ ). Our experiments seem to suggest that as $k \rightarrow 0$, the main contribution is proportional to the factor $\ln \left|1-e^{\frac{k \pi}{6} i}\right|$, and indeed it is easy to show that the $O(1)$ term is even uniformly bounded in $k$.

To obtain asymptotics for $S(T)$ when $k \rightarrow 0$ (and $T$ is bounded) is a bit more involved. We want to generalize [22, thm. 2.29, p. 468] by following the pointers provided in [22, proof of thm. 2.29, p. 468]. Basically, our goal is to single out any terms that grow as $k \rightarrow 0$ in the estimates for $S(T)$.

By careful bookkeeping we see that these terms are all of the form $\ln \left|1-e^{\frac{k \pi}{6} i}\right|$, and this kind of term only shows up when we apply the functional equation for the logarithmic derivative of the Selberg zeta function, $Z(s)$ ([22, thm. 2.18, p. 441]). To obtain the necessary estimate of $\frac{Z^{\prime}}{Z}(s)$ we study the integral on the left hand side of [21, prop. 8.6, p. 121]. Let the assumptions of [21, ass. 8.5 p. 120] and [21, p. 125 row 5] apply, e.g. $s=\sigma+i T, U=T+10$ and $\frac{1}{2} \leq \frac{1}{2}+\epsilon=\sigma_{1}<\sigma$. Through the integral estimates [21, prop. 8.7-8, 8.10-11] we consider either $\frac{Z^{\prime}}{Z}(\xi)-2 \ln \left|1-e^{\frac{k \pi}{6} i}\right|$ or $\frac{Z^{\prime}}{Z}(\xi)$, depending on whether the functional equation is applied along this part of the path or not, and then collect the extra terms at the end. It turns out that we consider the modified integrand in all parts to the left of $\Re(\xi)=\frac{1}{2}-\epsilon$. With this modification, all estimates in [21, prop. 8.7-8, 8.10-14, thm. 8.15 and 8.17] go through except for the addition of a term $R_{m}(s)=\frac{2}{\ln x} \ln \left|1-e^{\frac{k \pi}{6} i}\right| I_{m}(s)$, where $I_{m}(s)$ is the integral

$$
I_{m}(s)=\frac{1}{2 \pi i} \int_{\gamma} \frac{x^{\xi-s}-x^{2(\xi-s)}}{(\xi-s)^{2}} d \xi
$$

where the path is $\gamma=\gamma_{U}=\left[\frac{1}{2}-\epsilon-i U,-A-i U\right] \cup[-A-i U,-A+i U] \cup$ $\left[-A+i U, \frac{1}{2}-\epsilon+i U\right]$. The integrand has no poles to the right of $\Re(\xi)=\frac{1}{2}-\epsilon$ and is hence equal to the integral from $\frac{1}{2}-\epsilon-i U$ to $\frac{1}{2}-\epsilon+i U$. Elementary estimates now show that

$$
\left|I_{m}(s)\right| \leq \frac{2 x^{\left(\frac{1}{2}-\epsilon-\sigma\right)}}{2 \pi} \int_{-U}^{U} \frac{1}{\epsilon^{2}+(t-T)^{2}} d t \leq \frac{x^{-2 \epsilon}}{\pi \epsilon^{2}} \int_{-U}^{U} \frac{1}{1+\left(\frac{t-T}{\epsilon}\right)^{2}} d t \leq \frac{x^{-2 \epsilon}}{\epsilon} .
$$

Using $x=T^{\frac{1}{4}}$ (cf. [21, p. 138, row -6]) together with $\epsilon=\frac{1}{\ln x}$ (cf. [21, ass. 8.16(b), p. 130]) we see that

$$
\left|R_{m}(s)\right| \leq x^{-\frac{2}{\ln x}} \ln \left|1-e^{\frac{k \pi}{6} i}\right| \ln x \frac{1}{\ln x}=e^{-2} \ln \left|1-e^{\frac{k \pi}{6} i}\right| .
$$

This error term is now to be added to $\frac{Z^{\prime}}{Z}$ in [21, thm. 8.15] and subtracted from the right hand side of [21, thm. 8.17], and finally in [21, p. 135 (8.18)] we need to add $-\int_{\frac{1}{2}}^{2} \Im\left[R_{m}(s)\right]$ to the expression of $\pi S(T)$. Thus the analogue of [21, thm. 8.1, p. 119] and [22, thm. 2.29, p. 468] is $S(T)=O\left(\frac{T}{\ln T}\right)+O(1) \ln \left|1-e^{\frac{k \pi}{6} i}\right|$ 


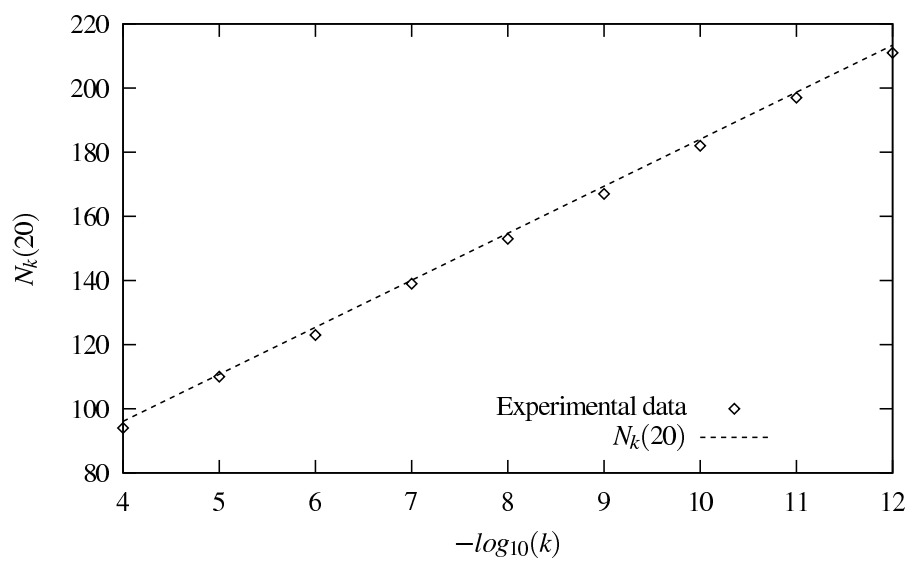

Figure 3. Plot of Weyl's law with constant $T=20$ and weight $k \rightarrow 0.1$

(with implied constants independent of $k$ ), and inserted into (8.1) we conclude the following Weyl's law:

$$
N_{k}(T)=\frac{T^{2}}{12}-\ln \left|1-e^{\frac{k \pi i}{6}}\right|\left[\frac{T}{\pi}+O(1)\right]+O\left(\frac{T}{\ln T}\right),
$$

uniformly for $0<k \leq 0.1$ (say) and $T \geq 2$, and the $O(1)$ term is bounded in magnitude by $\frac{3}{2 \pi} e^{-2} \approx 0.065$.

We have computed $N_{k}(T)$ experimentally for $k=10^{-j}, j=4, \ldots, 12$, and $0 \leq$ $T \leq 20$. Figure 3 shows a picture of the experimental values compared to the theoretical values obtained by (8.2) (with the $O$-terms neglected), and the difference indeed seems to be constant over this range.

From the form of Weyl's law above, we also see that the successive spacings $\Delta_{n}(k)=R_{n+1}(k)-R_{n}(k)$ should look almost like $\frac{1}{\frac{d N_{k}}{d T}}$, i.e. $\Delta_{n}(k) \approx \frac{1}{\frac{T}{6}+\frac{1}{\pi}\left|\ln \frac{k \pi}{6}\right|} \sim$ $\frac{\pi}{\left|\ln \frac{k \pi}{6}\right|}$ as $k \rightarrow 0$. Figure 4 provides a nice illustration of this fact, where it is clearly seen that the average spacings are almost constant for small $k$, and this constant is roughly proportional to $\frac{1}{|\ln k|}$.

The mean gap over 100 typical cases $\left(R_{n}, 5 \leq n \leq 155\right)$ turned out to be 0.117 at $k=10^{-9}, 0.107$ at $k=10^{-10}, 0.101$ at $k=10^{-11}$ and 0.095 at $k=10^{-12}$. The relative quotients, $\Delta_{n}(k)\left[\frac{R_{n}}{6}+\frac{1}{\pi}\left|\ln \frac{k \pi}{6}\right|\right]$, are 1.019, 0.990, 1.001 and 0.9934, respectively, and it is not inconceivable that one obtains 1 in the (logarithmic) limit.

8.2.1. Level repulsion. From figures such as Figure 4 one may be tempted to think that there are horizontal lines corresponding to cusp forms at weight 0 which cross the lines that are going down (i.e. corresponding to the Eisenstein series at weight $0)$. This is not the case! If we look closer we will see that there is actually "level repulsion" here, i.e. the horizontal "cusp-form-line" turns down before the "near crossing" and becomes an "Eisenstein-series-line", and the previous "Eisensteinseries-line" turns into a "cusp-form-line". See also Figures 5 and 6 , More precisely formulated: if there is a "near crossing" at the weight $k_{0}$ close to the eigenvalue $R_{0} \approx R_{j}\left(k_{0}\right) \approx R_{j+1}\left(k_{0}\right)$, then there are two analytic families $\phi_{j}(k)$ and $\phi_{j+1}(k)$ 


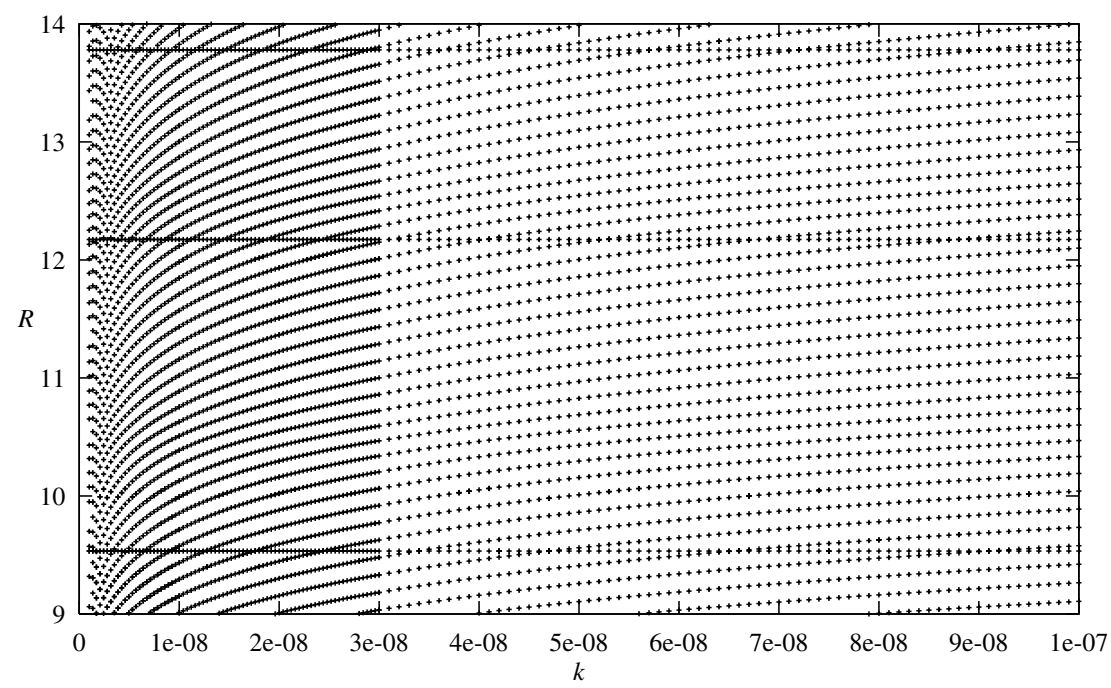

Figure 4. Plot of spectral parameters $R=R_{j}(k)$ with $(R, k) \in$ $[9,14] \times\left[10^{-9}, 10^{-7}\right]($ cf. Section 8.1$)$.

such that for some $\delta>\epsilon>0$,

$$
\phi_{j}(k) \in \begin{cases}C(k), & k \in\left[k_{0}+\epsilon, k_{0}+\delta\right], \\ E(k), & k \in\left[k_{0}-\delta, k_{0}-\epsilon\right],\end{cases}
$$

and

$$
\phi_{j+1}(k) \in \begin{cases}E(k), & k \in\left[k_{0}+\epsilon, k_{0}+\delta\right], \\ C(k), & k \in\left[k_{0}-\delta, k_{0}-\epsilon\right],\end{cases}
$$

and in the interval $\left(k_{0}-\epsilon, k_{0}+\epsilon\right)$ both families display a mixing between the two types $E(k)$ and $C(k)$. In fact, the Fourier coefficients of $\phi_{j+1}$ converge (as $k \rightarrow k_{0}-\epsilon$ ) to values close to the Fourier coefficients of $\phi_{j}$ for $k>k_{0}+\epsilon$ and vice versa. Since the two functions also need to be orthogonal, it is clear that the Fourier coefficients exhibit "wild" behavior in the small interval surrounding the "near crossing". Note also that, as $k \rightarrow 0$, all $\phi_{j}(k) \in E(k)$ and $R_{j}(k) \rightarrow 0$.

See Table 9 for examples of Fourier coefficients corresponding to eigenfunctions of types $E(k)$ and $C(k)$, close to an avoided crossing at weight $k=9.044605824 E-$ 8. Table 10 illustrates the agreement between the Fourier coefficients of a more generic cusp form in $E(k)$ and the corresponding coefficients of the Eisenstein series (appropriately normalized) at weight 0 . The level of agreement is striking to put it mildly; likewise in Table 9 for the $C(k)$ eigenfunction. The "1 for 1" nature of this convergence in the presence of a limiting continuous spectrum seems not to have been suspected earlier. Cf. [24, thm. 6.6 and cor. 6.9] and 23].

The fact that the system seems to avoid accidental degeneracies by means of level repulsion and avoided crossings is in agreement with the Wigner-von Neumann theorem; cf. [72].

8.3. Lifts at weight 1. As we saw in Section 4.5.2 we could prove the existence of certain Hecke relations at weight 1 (e.g. (4.19), (4.20) and 4.21)). Tables 2 and 3 contain numerical verifications of these relations. Table 4 contains a list of 

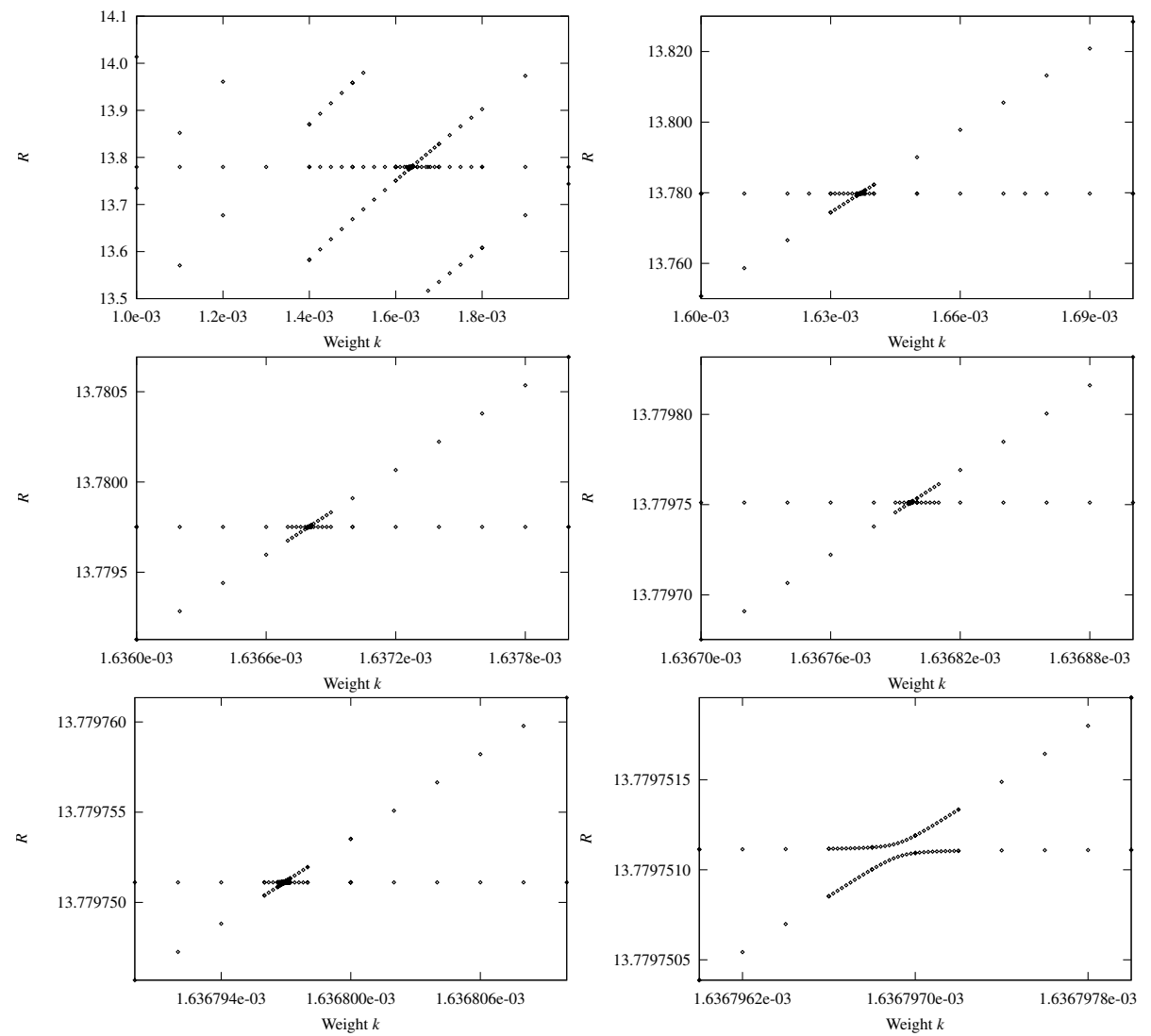

FiguRE 5. Level repulsion at $R=13.779 \ldots$

computed eigenvalues on $\mathcal{M}\left(\Gamma_{0}(1), v_{\eta}^{2}, 1, \lambda\right)$, and the eigenvalues corresponding to cosine CM-forms are indicated. In these cases, we have computed the actual error since we know the exact eigenvalues:

$$
R_{k}=\frac{2 \pi k}{\ln (7+2 \sqrt{12})}, k \in \mathbb{Z}^{+} .
$$

Note that the actual error is in general much smaller than the error-parameter which is basically $H\left(Y_{1}, Y_{2}\right)=\left|c(2)-c^{\prime}(2)\right|+\left|c(3)-c^{\prime}(3)\right|+\left|c(4)-c^{\prime}(4)\right|$, where $c(n)$ is computed with $Y_{1}$ and $c^{\prime}(n)$ with $Y_{2}$.

8.4. Half integer weight. We now consider the case of $\Gamma_{0}(4)$ and the $\theta$-multiplier system for weight $k=\frac{1}{2}$. The aim of our investigation in this case was to study the Shimura lift, and in particular to investigate the dimensions of the spaces of half integer weight forms. As remarked at the end of Section 6 several properties of the Shimura correspondence were observed numerically, and in the original version of these notes, [64, ch. 2], we formulated a number of experimentally inspired conjectures. Except for the question of injectivity, these conjectures have now been resolved with Propositions 5 and 6 . (With the obvious correction in the first conjecture: the dimension of $\mathcal{M}\left(\Gamma_{0}(4), v_{\theta}, \frac{1}{2}, R\right)$ corresponding to an oldspace is at least two dimensional.) 

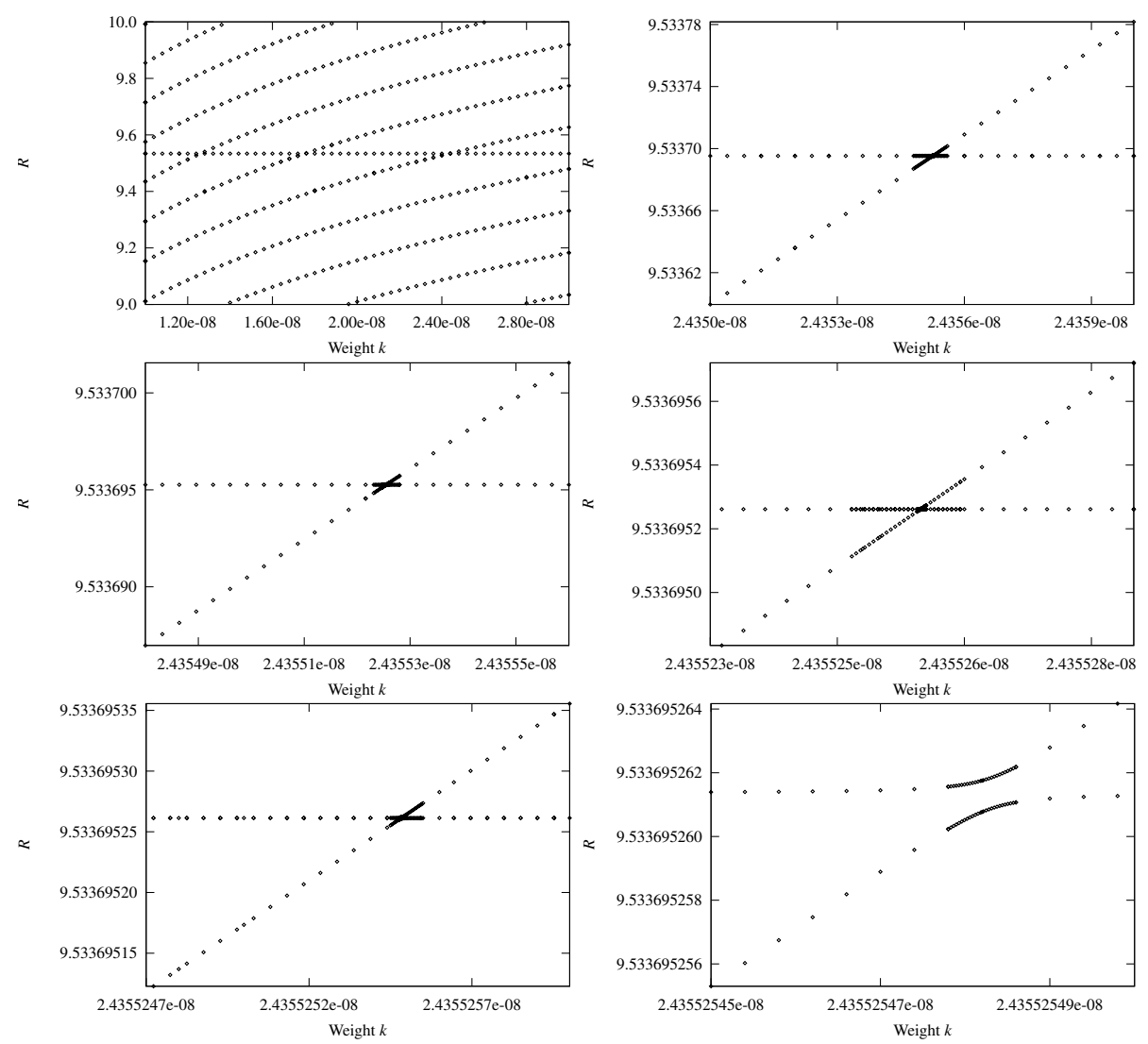

FiguRE 6 . Level repulsion at $R=9.533 \ldots$.

Tables 5 and 6 contain examples of Fourier coefficients at weight $\frac{1}{2}$. Note especially in Table 6 the agreement with Proposition 6 c) displayed by the Fourier coefficients $c(n)$ for $n \equiv 5,1 \bmod 8$ respectively. Here, it is known that 8.92287648699174 and 12.09299487507860 on $\Gamma_{0}(2)$ correspond to the eigenvalues 1 and -1 , respectively, with respect to the involution $\omega_{2}$.

Table 8 contains a comparison of Fourier coefficients computed both from forms on $\Gamma_{0}(2)$ via (6.1) and computed directly. Additional Fourier coefficients for the weight 0 forms are available in Table 7 
TABLE 1. Eigenvalues for $N=1, v=v_{\eta}$

\begin{tabular}{|c|c|c|c|c|c|}
\hline \multirow[t]{2}{*}{$k=5.0$} & \multicolumn{5}{|c|}{$k=5.25$} \\
\hline & $|c(-1)|^{\mathrm{a}}$ & $H(y 1, y 2)$ & $R$ & $|c(-1)|^{\mathrm{a}}$ & $H(y 1, y 2)$ \\
\hline 3.66240686691 & $2 \mathrm{E}+3$ & 8E-09 & 3.68037312372 & $3 \mathrm{E}+3$ & $3 \mathrm{E}-08$ \\
\hline 5.77698688079 & $6 \mathrm{E}+3$ & $1 \mathrm{E}-09$ & 5.82067054942 & $9 \mathrm{E}+3$ & $6 \mathrm{E}-09$ \\
\hline 6.64285171609 & $1 \mathrm{E}+4$ & $2 \mathrm{E}-09$ & 6.63460520751 & $2 \mathrm{E}+4$ & $3 \mathrm{E}-09$ \\
\hline 7.82634704661 & $7 \mathrm{E}+4$ & $8 \mathrm{E}-07$ & 7.90867228426 & $2 \mathrm{E}+5$ & $9 \mathrm{E}-09$ \\
\hline 8.66620831839 & $8 \mathrm{E}+4$ & $1 \mathrm{E}-08$ & 8.61646891946 & $1 \mathrm{E}+5$ & $8 \mathrm{E}-09$ \\
\hline 9.45156176778 & $4 \mathrm{E}+4$ & $4 \mathrm{E}-09$ & 9.56930344151 & $7 \mathrm{E}+4$ & $5 \mathrm{E}-09$ \\
\hline 10.21802876612 & $9 \mathrm{E}+4$ & $2 \mathrm{E}-08$ & 10.15656706121 & $2 \mathrm{E}+5$ & $2 \mathrm{E}-09$ \\
\hline 10.65897262925 & $2 \mathrm{E}+5$ & $5 \mathrm{E}-09$ & 10.70911890024 & $2 \mathrm{E}+5$ & $4 \mathrm{E}-10$ \\
\hline 11.27526358329 & $2 \mathrm{E}+5$ & $2 \mathrm{E}-08$ & 11.34046324165 & $4 \mathrm{E}+5$ & $2 \mathrm{E}-08$ \\
\hline 12.15792337439 & $5 \mathrm{E}+6$ & $2 \mathrm{E}-06$ & 12.11839521329 & $4 \mathrm{E}+6$ & $2 \mathrm{E}-06$ \\
\hline 12.55403510011 & $3 \mathrm{E}+5$ & $3 \mathrm{E}-09$ & 12.65021958486 & $4 \mathrm{E}+5$ & $9 \mathrm{E}-09$ \\
\hline 13.00123950671 & $4 \mathrm{E}+4$ & $2 \mathrm{E}-09$ & 13.02622821839 & $8 \mathrm{E}+4$ & $2 \mathrm{E}-09$ \\
\hline 13.67542640619 & $8 \mathrm{E}+5$ & $8 \mathrm{E}-09$ & 13.56022943627 & $1 \mathrm{E}+6$ & $1 \mathrm{E}-07$ \\
\hline 13.71353384347 & $4 \mathrm{E}+6$ & $4 \mathrm{E}-07$ & 13.87057635696 & $7 \mathrm{E}+5$ & $4 \mathrm{E}-07$ \\
\hline 14.47039277248 & $6 \mathrm{E}+5$ & $1 \mathrm{E}-09$ & 14.48204838116 & $1 \mathrm{E}+6$ & $7 \mathrm{E}-05$ \\
\hline 15.03845367363 & $1 \mathrm{E}+6$ & $6 \mathrm{E}-09$ & 15.09966704087 & $4 \mathrm{E}+7$ & $1 \mathrm{E}-07$ \\
\hline 15.39856858318 & $1 \mathrm{E}+6$ & $1 \mathrm{E}-09$ & 15.38981845044 & $1 \mathrm{E}+6$ & $4 \mathrm{E}-09$ \\
\hline 15.85705128856 & $7 \mathrm{E}+4$ & $7 \mathrm{E}-09$ & 15.94059443942 & $1 \mathrm{E}+4$ & $3 \mathrm{E}-09$ \\
\hline 16.14536205683 & $5 \mathrm{E}+6$ & $2 \mathrm{E}-07$ & 16.09999759486 & $8 \mathrm{E}+6$ & $7 \mathrm{E}-06$ \\
\hline 16.45061260141 & $2 \mathrm{E}+6$ & $1 \mathrm{E}-08$ & 16.52671557073 & $5 \mathrm{E}+6$ & $1 \mathrm{E}-09$ \\
\hline 16.93043847901 & $2 \mathrm{E}+7$ & $2 \mathrm{E}-08$ & 16.90856097808 & $2 \mathrm{E}+7$ & $5 \mathrm{E}-08$ \\
\hline 17.51562192888 & $2 \mathrm{E}+5$ & $4 \mathrm{E}-09$ & 17.53730159778 & $1 \mathrm{E}+6$ & $3 \mathrm{E}-10$ \\
\hline 17.59022138300 & $1 \mathrm{E}+6$ & $6 \mathrm{E}-10$ & 17.74142373355 & $7 \mathrm{E}+6$ & $4 \mathrm{E}-09$ \\
\hline 18.13826107361 & $7 \mathrm{E}+6$ & $2 \mathrm{E}-08$ & 18.02022951826 & $6 \mathrm{E}+6$ & $9 \mathrm{E}-10$ \\
\hline 18.32637702289 & $5 \mathrm{E}+5$ & $5 \mathrm{E}-09$ & 18.37970066644 & $8 \mathrm{E}+5$ & $2 \mathrm{E}-09$ \\
\hline 18.76341585136 & $2 \mathrm{E}+6$ & $5 \mathrm{E}-09$ & 18.90587158951 & $1 \mathrm{E}+7$ & $8 \mathrm{E}-09$ \\
\hline 19.16629116326 & $4 \mathrm{E}+6$ & $8 \mathrm{E}-10$ & 19.09726131554 & $1 \mathrm{E}+7$ & $5 \mathrm{E}-10$ \\
\hline 19.67214438521 & $3 \mathrm{E}+7$ & $2 \mathrm{E}-07$ & 19.66894569593 & $5 \mathrm{E}+6$ & $3 \mathrm{E}-10$ \\
\hline 19.68520099819 & $1 \mathrm{E}+6$ & $3 \mathrm{E}-10$ & 19.73195996101 & $7 \mathrm{E}+6$ & $4 \mathrm{E}-10$ \\
\hline 20.00524829746 & $2 \mathrm{E}+6$ & $4 \mathrm{E}-09$ & 20.12609436572 & $2 \mathrm{E}+6$ & $3 \mathrm{E}-10$ \\
\hline 20.38266630653 & $3 \mathrm{E}+6$ & $3 \mathrm{E}-10$ & 20.35571778301 & $1 \mathrm{E}+7$ & $7 \mathrm{E}-10$ \\
\hline 20.67297062056 & $6 \mathrm{E}+6$ & $4 \mathrm{E}-08$ & 20.71020380483 & $6 \mathrm{E}+6$ & $8 \mathrm{E}-09$ \\
\hline 20.97339376061 & $6 \mathrm{E}+6$ & $8 \mathrm{E}-10$ & 20.88321504381 & $1 \mathrm{E}+7$ & $2 \mathrm{E}-09$ \\
\hline
\end{tabular}

a The normalization we have used here is the usual $c(1)=1$. 
TABle 2. Fourier coefficients for a CMform $f \in \mathcal{M}\left(\Gamma_{0}(1), v_{\eta}^{2}, 1,4.770984191561\right)$

\begin{tabular}{|c|c|c|c|}
\hline$n$ & & $c(n) / c(0)^{\mathrm{a}}$ & Error \\
\hline 0 & 1.755930576575 & & \\
\hline 1 & 1.000000000000 & & \\
\hline 2 & -1.755930576574 & $c(27)$ & $0.4 \mathrm{E}-08$ \\
\hline 3 & 1.571810322167 & $c(40)$ & $0.1 \mathrm{E}-08$ \\
\hline 4 & 1.755930576575 & $c(53)$ & $0.7 \mathrm{E}-08$ \\
\hline 5 & -1.770268323978 & $c(66)$ & $0.3 \mathrm{E}-09$ \\
\hline 6 & -2.474798320759 & $c(79)$ & $0.1 \mathrm{E}-08$ \\
\hline 7 & 0.000000000000 & $c(92)$ & $0.4 \mathrm{E}-08$ \\
\hline 8 & 0.346240855507 & $c(105)$ & $0.5 \mathrm{E}-08$ \\
\hline 9 & 3.510179255561 & $c(118)$ & $0.3 \mathrm{E}-08$ \\
\hline 10 & 1.116593241680 & $c(131)$ & $0.4 \mathrm{E}-08$ \\
\hline 11 & -0.000000000001 & $c(144)$ & $0.3 \mathrm{E}-08$ \\
\hline 12 & 0.000000000001 & $c(157)$ & $0.1 \mathrm{E}-07$ \\
\hline 13 & -3.019229958496 & $c(170)$ & $0.8 \mathrm{E}-08$ \\
\hline 14 & -1.186431979458 & $c(183)+c(1)$ & $0.3 \mathrm{E}-08$ \\
\hline 15 & -3.079783541463 & $\begin{array}{c}c(196) \\
-c(n) c(-1) / R^{2} / c(0)\end{array}$ & $0.5 \mathrm{E}-08$ \\
\hline-1 & 5.055064268188 & $\mathrm{~b}$ & $0.1 \mathrm{E}-11$ \\
\hline-2 & -11.180067729976 & $c(21)$ & $0.4 \mathrm{E}-07$ \\
\hline-3 & 0.000000000001 & $c(32)$ & $0.2 \mathrm{E}-07$ \\
\hline-4 & 16.472675660354 & $c(43)$ & $0.2 \mathrm{E}-08$ \\
\hline-5 & 16.729030199659 & $c(54)$ & $0.5 \mathrm{E}-07$ \\
\hline-6 & 13.098490835617 & $c(65)$ & $0.5 \mathrm{E}-08$ \\
\hline-7 & 16.046414105740 & $c(76)$ & $0.1 \mathrm{E}-07$ \\
\hline-8 & -0.000000000023 & $c(87)$ & $0.1 \mathrm{E}-07$ \\
\hline-9 & 13.340740291248 & $c(98)$ & $0.3 \mathrm{E}-07$ \\
\hline-10 & 0.000000000006 & $c(109)$ & $0.4 \mathrm{E}-07$ \\
\hline-11 & 2.151215034503 & $c(120)$ & $0.7 \mathrm{E}-08$ \\
\hline-12 & 2.878852009050 & $c(131)-c(1)$ & $0.2 \mathrm{E}-07$ \\
\hline-13 & 0.000000000039 & $c(142)$ & $0.6 \mathrm{E}-08$ \\
\hline-14 & 7.496795049955 & $c(153)$ & $0.4 \mathrm{E}-07$ \\
\hline-15 & -12.830881007618 & $c(164)$ & $0.4 \mathrm{E}-07$ \\
\hline
\end{tabular}


TABLE 3. Fourier coefficients for a non-CM-form $f \in$ $\mathcal{M}\left(\Gamma_{0}(1), v_{\eta}^{2}, 1,3.66240686698667\right)$

\begin{tabular}{rrcc}
$n$ & & $c(n) / c(0)^{\mathrm{a}}$ & Error \\
\hline 0 & -1.352193685534 & & \\
1 & 1.000000000000 & & \\
2 & -1.697113317091 & $c(27)$ & $0.5 \mathrm{E}-08$ \\
3 & -0.057989599353 & $c(40)$ & $0.4 \mathrm{E}-07$ \\
4 & 2.461764397786 & $c(53)$ & $0.1 \mathrm{E}-07$ \\
5 & 0.510856433057 & $c(66)$ & $0.6 \mathrm{E}-08$ \\
6 & -0.952325903762 & $c(79)$ & $0.8 \mathrm{E}-08$ \\
7 & -1.660630683908 & $c(92)$ & $0.2 \mathrm{E}-07$ \\
8 & -2.343382246022 & $c(105)$ & $0.9 \mathrm{E}-08$ \\
9 & 1.271097206907 & $c(118)$ & $0.1 \mathrm{E}-07$ \\
10 & -0.203512820511 & $c(131)$ & $0.6 \mathrm{E}-08$ \\
11 & 2.110622602834 & $c(144)$ & $0.5 \mathrm{E}-08$ \\
12 & 2.170616908700 & $c(157)$ & $0.4 \mathrm{E}-08$ \\
13 & 0.449799127363 & $c(170)$ & $0.4 \mathrm{E}-07$ \\
14 & 0.612654661780 & $c(183)+c(1)$ & $0.4 \mathrm{E}-07$ \\
15 & -1.684453740441 & $c(196)$ & $0.1 \mathrm{E}-07$ \\
16 & 0.400312170289 & $c(209)$ & $0.2 \mathrm{E}-07$ \\
17 & -2.868395110060 & $c(222)$ & $0.1 \mathrm{E}-07$ \\
18 & -1.931595991172 & $c(235)$ & $0.2 \mathrm{E}-07$ \\
19 & -0.591212766919 & $c(248)$ & $0.1 \mathrm{E}-07$ \\
20 & 0.792151138999 & $c(261)$ & $0.9 \mathrm{E}-08$ \\
21 & -1.717242193922 & $c(274)$ & $0.3 \mathrm{E}-07$ \\
22 & 1.369169138277 & $c(287)$ & $0.2 \mathrm{E}-07$ \\
23 & 2.007854712832 & $c(300)$ & $0.8 \mathrm{E}-09$ \\
24 & 0.447826147902 & $c(313)$ & $0.1 \mathrm{E}-07$ \\
25 & 3.051006373828 & $c(326)$ & $0.4 \mathrm{E}-08$ \\
26 & -0.032419986064 & $c(339)$ & $0.6 \mathrm{E}-08$ \\
27 & 1.255081531820 & $c(352)+a(2)$ & $0.2 \mathrm{E}-07$ \\
28 & -0.707047087424 & $c(365)$ & $0.3 \mathrm{E}-07$ \\
29 & 1.272283355260 & $c(378)$ & $0.1 \mathrm{E}-07$ \\
30 & -0.184187214400 & $c(391)$ & $0.2 \mathrm{E}-07$
\end{tabular}

a This quotient is deduced from formula (4.16) or (4.17) on p. 2390. 
TABLE 4. Eigenvalues for $\mathcal{M}\left(P S L(2, \mathbb{Z}), 1, v_{\eta}^{2}\right)$

\begin{tabular}{|c|c|c|}
\hline$R$ & $H\left(y_{1}, y_{2}\right)$ & True error $^{\mathrm{a}}$ \\
\hline 2.38549209578045 & $1 \mathrm{E}-12$ & $1 \mathrm{E}-15^{\mathrm{b}}$ \\
\hline 3.66240686698667 & $1 \mathrm{E}-11$ & \\
\hline 4.77098419156091 & $1 \mathrm{E}-13$ & $1 \mathrm{E}-14^{\mathrm{b}}$ \\
\hline 5.77698688078694 & $6 \mathrm{E}-12$ & \\
\hline 6.64285171613711 & $1 \mathrm{E}-11$ & \\
\hline 7.15647628734173 & $1 \mathrm{E}-11$ & $4 \mathrm{E}-13^{\mathrm{b}}$ \\
\hline 7.82634704540775 & $1 \mathrm{E}-13$ & \\
\hline 8.66620831896793 & $7 \mathrm{E}-14$ & \\
\hline 9.45156176783224 & $7 \mathrm{E}-12$ & \\
\hline 9.54196838312186 & $2 \mathrm{E}-13$ & $6 \mathrm{E}-14^{\mathrm{b}}$ \\
\hline 10.21802876776059 & $3 \mathrm{E}-13$ & \\
\hline 10.65897262920241 & $2 \mathrm{E}-12$ & \\
\hline 11.27526358349387 & $2 \mathrm{E}-13$ & \\
\hline 11.92746047890219 & $7 \mathrm{E}-11$ & $5 \mathrm{E}-14^{\mathrm{b}}$ \\
\hline 12.15792337422149 & $7 \mathrm{E}-13$ & \\
\hline 12.55403509998720 & $1 \mathrm{E}-13$ & \\
\hline 13.00123950642372 & $9 \mathrm{E}-13$ & \\
\hline 13.67542640643589 & $2 \mathrm{E}-13$ & \\
\hline 13.71353384358095 & $1 \mathrm{E}-12$ & \\
\hline 14.31295257468268 & $1 \mathrm{E}-12$ & $1 \mathrm{E}-14^{\mathrm{b}}$ \\
\hline 14.47039277253940 & $1 \mathrm{E}-12$ & \\
\hline 15.03845367358721 & $2 \mathrm{E}-13$ & \\
\hline 15.39856858348441 & $1 \mathrm{E}-12$ & \\
\hline 15.85705128717333 & $3 \mathrm{E}-10$ & \\
\hline 16.14536205734475 & $3 \mathrm{E}-11$ & \\
\hline 16.45061260131967 & $4 \mathrm{E}-11$ & \\
\hline 16.69844467046304 & $4 \mathrm{E}-12$ & $1 \mathrm{E}-13^{\mathrm{a}}$ \\
\hline 16.93043847896222 & $4 \mathrm{E}-15$ & \\
\hline 17.51562192885174 & $2 \mathrm{E}-10$ & \\
\hline 17.59022138305996 & $1 \mathrm{E}-10$ & \\
\hline 18.13826107340244 & $3 \mathrm{E}-11$ & \\
\hline 18.32637702205910 & $4 \mathrm{E}-11$ & \\
\hline 18.76341585146817 & $1 \mathrm{E}-11$ & \\
\hline
\end{tabular}


TABLE 5. Fourier coefficients for $f_{1,2} \in \mathcal{M}\left(\Gamma_{0}(4), \frac{1}{2}, 6.889875675945\right)$

Fourier coefficients for $f_{1} \in V^{+}$; observe that $a(n)=0$ for $n \equiv 2,3 \bmod 4$

\begin{tabular}{rr}
$n$ & \multicolumn{1}{c}{$a(n)$} \\
\hline 4 & 0.84219769675471 \\
5 & 0.18355821406443 \\
8 & 0.56907998524429 \\
9 & -0.33045049673565 \\
12 & -0.41296169213831 \\
13 & 0.60153537988057 \\
16 & 0.30482066289397 \\
17 & -0.88689598690620 \\
20 & 0.41418282092059 \\
21 & 0.50212917023175 \\
23 & -0.00000000000110 \\
24 & -1.04429548341249 \\
25 & 0.28984678984391
\end{tabular}

\begin{tabular}{rr}
\multicolumn{1}{c}{$n$} & \multicolumn{1}{c}{$a(-n)$} \\
\hline 3 & 1.01825299171456 \\
4 & 2.18968040385979 \\
7 & 2.06305218095270 \\
8 & -1.17157116610978 \\
11 & -1.02718719694121 \\
12 & 2.29759751514531 \\
15 & -4.08935474990375 \\
16 & 3.39248165496032 \\
19 & -1.31804633824673 \\
20 & 1.87725570455517 \\
23 & -2.22265197818114 \\
24 & 0.58816620330140
\end{tabular}

$|c(4) c(9)-c(36)|=0.2 E-08$

Fourier coefficients for $f_{2} \notin V^{+}$; observe that $a(n)=0$ for $n \equiv 1 \bmod 8$

\begin{tabular}{rr}
$n$ & \multicolumn{1}{c}{$a(n)$} \\
\hline 0 & 0.000000000000 \\
1 & 0.000000000000 \\
2 & 1.000000000000 \\
3 & -0.725665465042 \\
4 & -0.710754741008 \\
5 & 0.456158224759 \\
6 & -1.835059236817 \\
7 & 0.481289183972 \\
8 & 0.707106781187 \\
9 & 0.000000000000 \\
10 & -0.651049038069 \\
11 & -0.470914040036 \\
12 & -0.513122971204 \\
13 & 1.494868058150 \\
14 & 0.968484734380 \\
15 & -0.945563574521 \\
16 & -1.101175502961 \\
17 & 0.000000000000 \\
18 & 0.824250041644
\end{tabular}

\begin{tabular}{cr}
$n$ & \multicolumn{1}{c}{$a(n)$} \\
\hline 19 & -0.936283350934 \\
20 & 0.192087703124 \\
21 & 1.247834928335 \\
22 & 0.411443268212 \\
23 & -0.018564690418 \\
24 & -1.297582830232 \\
25 & 0.000000000000 \\
26 & -0.610656711780 \\
27 & -0.179166638197 \\
28 & 0.340322845698 \\
29 & 0.001563849679 \\
30 & 1.462745418892 \\
31 & 0.400763051265 \\
32 & 0.095523702475 \\
33 & 0.000000000000 \\
34 & -0.879782299796 \\
35 & -1.833603623066 \\
36 & 0.234869257223
\end{tabular}


TABLE 6. Fourier coefficients for $f \in \mathcal{M}\left(\Gamma_{0}(4), \frac{1}{2}, R\right)$

\begin{tabular}{rr}
\multicolumn{2}{c}{$R=4.461438243496$} \\
$n$ & $a(n)$ \\
\hline 0 & 0.00000000000000 \\
1 & 1.00000000000000 \\
2 & 0.63334968449036 \\
3 & 0.63517832947402 \\
4 & -0.70710678118667 \\
5 & -0.00000000000003 \\
6 & 1.28035706400142 \\
7 & -0.90756258916698 \\
8 & -0.44784585676555 \\
9 & 0.52643872643776 \\
10 & -0.57763498966972 \\
11 & 1.12485377915641 \\
12 & -0.44913890403389 \\
13 & 0.00000000000001 \\
14 & 0.48078071833327 \\
15 & -1.58539012005784 \\
16 & 0.50000000000015 \\
17 & -0.14882069214483 \\
18 & 1.06474902295605 \\
19 & 0.21268916863632 \\
20 & 0.00000000000003 \\
21 & 0.00000000000002 \\
22 & -1.56248056455209 \\
23 & 0.85478501960318 \\
24 & -0.90534916229569 \\
25 & 0.45696099733973 \\
36 & -0.37224839333969 \\
\hline$|c(4) c(9)-c(36)|=4 E-12$ \\
& $|c(21)|=2 E-14$ \\
&
\end{tabular}

\begin{tabular}{rr}
\multicolumn{3}{c}{$R=6.046497437542$} \\
$n$ & $a(n)$ \\
\hline 0 & 0.000000000000 \\
1 & 0.000000000000 \\
2 & 1.000000000000 \\
3 & 1.770795863371 \\
4 & 0.000000000029 \\
5 & 1.331470494003 \\
6 & -0.749395507674 \\
7 & 0.074369313625 \\
8 & 0.707106781209 \\
9 & -0.000000000016 \\
10 & -1.214451367832 \\
11 & 0.620756243833 \\
12 & 1.252141763204 \\
13 & -1.123686021586 \\
14 & -0.711286031630 \\
15 & 0.964706032413 \\
16 & -0.000000000112 \\
17 & -0.000000000108 \\
18 & -0.128644484609 \\
19 & -0.499664871594 \\
20 & 0.941491814843 \\
21 & -0.447011950327 \\
22 & -0.805501416928 \\
23 & 1.236716356721 \\
24 & -0.529902643583 \\
25 & 0.000000008756 \\
36 & -0.000000000168 \\
288 & -0.064322242377 \\
\hline$c\left(2 \cdot 3^{2}\right) c\left(2 \cdot 4^{2}\right)-c\left(2 \cdot 12^{2}\right) \mid=8 E-11$ \\
& $|c(17)|=1 E-10$ \\
\hline &
\end{tabular}


TABLE 7. Supplemental table of Fourier coefficients for $\mathcal{M}\left(\Gamma_{0}(2), 0, R\right)$

\begin{tabular}{rrrrrr}
$R$ & 8.92287648699174 & & $R$ & 12.0929948750786 \\
$n$ & $A(n)$ & & $n$ & $A(n)$ \\
\cline { 1 - 2 } \cline { 5 - 6 } 2 & -0.70710678118654 & & 2 & 0.70710678118655 \\
3 & 1.10378899562734 & & 3 & -0.70599475399569 \\
4 & 0.49999999999993 & & 4 & 0.49999999999999 \\
5 & 0.90417459283958 & & 5 & -0.79974825694039 \\
6 & -0.78049668380711 & & 6 & -0.49921367803249 \\
7 & 0.82934246755499 & & 7 & -1.71337067862845 \\
8 & -0.35355339059330 & & 8 & 0.35355339059328 \\
9 & 0.21835014686776 & & 9 & -0.50157140733054 \\
10 & -0.63934798597339 & & 10 & -0.56550741572467 \\
\hline$R$ & 13.77975135189073 & & $R$ & 13.77975135189073 \\
& $\left(\right.$ even wrt $\left.z \mapsto-\frac{1}{2 z}\right)$ & & & $\left(\right.$ odd wrt $\left.z \mapsto-\frac{1}{2 z}\right)$ \\
$n$ & $A(n)$ & & $n$ & \\
2 & 2.96351804031448 & & 2 & 0.13509091556820 \\
3 & 0.24689977245401 & & 3 & 0.24689977245398 \\
4 & 3.59139177031902 & & 4 & -0.79070303958101 \\
5 & 0.73706038534834 & & 5 & 0.73706038534830 \\
6 & 0.73169192981688 & & 6 & 0.03335391631439 \\
7 & -0.26142007576500 & & 7 & -0.26142007576538 \\
8 & 2.60064131148226 & & 8 & -1.36013067551284 \\
9 & -0.93904050235826 & & 9 & -0.93904050236089 \\
10 & 2.18429174878080 & & 10 & 0.09957016228575
\end{tabular}

TABLE 8. Comparison of Fourier coefficients $A(n)$ computed directly for $\mathcal{M}\left(\Gamma_{0}(N), 0, R\right)(N=1,2)$, vs. $\hat{A}(n)$ computed on $\mathcal{M}\left(\Gamma_{0}(4), \frac{1}{2}, \frac{1}{2} R\right)$ and using (6.1)

\begin{tabular}{|c|c|c|c|}
\hline$n$ & $\hat{A}(n)$ & $A(n)$ & $|A(n)-\hat{A}(n)|$ \\
\hline \multicolumn{4}{|c|}{$f \in \mathcal{M}\left(\Gamma_{0}(2), 0,8.922876486992\right)(t=1)$} \\
\hline 2 & -0.70710678118665 & -0.707106781186 & $0.6 \mathrm{E}-12$ \\
\hline 3 & 1.10378899562739 & 1.103788995627 & $0.4 \mathrm{E}-12$ \\
\hline 5 & 0.90417459283969 & 0.904174592840 & $0.3 \mathrm{E}-12$ \\
\hline \multicolumn{4}{|c|}{$f \subseteq \mathcal{M}\left(\Gamma_{0}(1), 0,13.779751351891\right)(t=1)$} \\
\hline 2 & 1.54930447794126 & 1.549304477941 & $0.7 \mathrm{E}-12$ \\
\hline 3 & 0.24689977245398 & 0.246899772454 & $0.3 \mathrm{E}-12$ \\
\hline 4 & 1.40034436536892 & 1.400344365369 & $0.2 \mathrm{E}-12$ \\
\hline 5 & 0.73706038534387 & 0.737060385348 & $0.4 \mathrm{E}-11$ \\
\hline 6 & 0.38252292109716 & 0.382522923066 & $0.2 \mathrm{E}-08$ \\
\hline \multicolumn{4}{|c|}{$f \in \mathcal{M}\left(\Gamma_{0}(1), 0,13.779751351891\right)(t=2)$} \\
\hline 3 & 0.24689977245437 & 0.246899772454 & $0.8 \mathrm{E}-13$ \\
\hline 5 & 0.73706038535004 & 0.737060385348 & $0.2 \mathrm{E}-11$ \\
\hline 7 & -0.26142007624377 & -0.261420075765 & $0.5 \mathrm{E}-09$ \\
\hline 9 & -0.93904050238904 & -0.939040502362 & $0.3 \mathrm{E}-10$ \\
\hline \multicolumn{4}{|c|}{$f \in \mathcal{M}\left(\Gamma_{0}(2), 0,12.092994875079\right)(t=2)$} \\
\hline 3 & -0.70599475379863 & -0.705994753996 & $0.2 \mathrm{E}-09$ \\
\hline 5 & -0.79974825694696 & -0.799748256940 & $0.7 \mathrm{E}-11$ \\
\hline 7 & -1.71337067860377 & -1.713370678628 & $0.2 \mathrm{E}-10$ \\
\hline 9 & -0.50157140750090 & -0.501571407330 & $0.2 \mathrm{E}-09$ \\
\hline
\end{tabular}


TABle 9. Comparison of Fourier coefficients for weights $k=$ $9.044605824 E-08$ and $k=0$ near an "avoided crossing"

Corresponds to the cusp form

$\begin{array}{crll}k & 9.0446058240 \mathrm{E}-08 & 0 & \text { Difference } \\ R & 13.77975135189074 & 13.77975135189074 & \\ c(2) & 1.54930480559976 & 1.54930447794069 & 0.3 \mathrm{E}-06 \\ c(3) & 0.24689988546553 & 0.24689977245411 & 0.1 \mathrm{E}-06 \\ c(4) & 1.40034433555250 & 1.40034436536841 & 0.1 \mathrm{E}-06 \\ c(5) & 0.73706067260516 & 0.73706038534787 & 0.2 \mathrm{E}-06 \\ c(6) & 0.38252272069428 & 0.38252292306557 & 0.2 \mathrm{E}-06\end{array}$

Corresponds to the Eisenstein series

$\begin{array}{crrr}k & 9.0446058240 \mathrm{E}-08 & 0 & \text { Difference } \\ R & 13.77975135138225 & 13.77975135138225 & \\ c(2) & -2.06525760334129 & -1.98398933080188 & 0.8 \mathrm{E}-01 \\ c(3) & -1.72891679536648 & -1.68449330640991 & 0.4 \mathrm{E}-01 \\ c(4) & 2.97153986917404 & 2.93621366473571 & 0.4 \mathrm{E}-01 \\ c(5) & -2.02747287754385 & -1.96531634618530 & 0.6 \mathrm{E}-01 \\ c(6) & 3.41008729668221 & 3.34201674772446 & 0.7 \mathrm{E}-01\end{array}$

The coefficients for the Eisenstein series at $k=0$ were computed using (5.1), i.e., $c(2)=2 \cos (R \ln 2)$

$c(3)=2 \cos (R \ln 3)$

$c(4)=1+2 \cos (R \ln 4)$

$c(5)=2 \cos (R \ln 5)$

$c(6)=2 \cos (R \ln 6)+2 \cos (R(\ln 3-\ln 2))$

TABLE 10. Comparison of Fourier coefficients for weights $k=$ $9.044605824 E-08$ and $k=0$ "far" from an "avoided crossing". The weight 0 coefficients were computed using the formulas in Table 9

$\begin{array}{crrr}k & 9.0446058240 \mathrm{E}-08 & 0 & \text { Difference } \\ R & 13.62696884857618 & 13.62696884857618 & \\ c(2) & -1.99957085683552 & -1.99957081810438 & 0.4 \mathrm{E}-07 \\ c(3) & -1.48069687587703 & -1.48069680342062 & 0.7 \mathrm{E}-07 \\ c(4) & 2.99828354611637 & 2.99828345661464 & 0.7 \mathrm{E}-07 \\ c(5) & -1.99647405201235 & -1.99647406885962 & 0.2 \mathrm{E}-07 \\ c(6) & 2.96075820067617 & 2.96075811858031 & 0.8 \mathrm{E}-07\end{array}$




\section{ACKNOWLEDGMENTS}

This paper is based on Chapter 2 of my Ph.D. thesis 64 but with additional theoretical discussions, e.g., in Sections 6 and 4.5, I am grateful to my advisor Dennis Hejhal and my second advisor Andreas Strm̈bergsson for many giving discussions and many valuable comments and suggestions.

\section{REFERENCES}

1. T. M. Apostol, Modular Functions and Dirichlet Series in Number Theory, Springer-Verlag, 1976.

2. T. Arakawa, Shimura correspondence for Maass wave forms and Selberg zeta functions, Proceedings of the conference "Automorphic forms and representations of algebraic groups over local fields" (Hiroshi Saito and Tetusya Takahashi, eds.), RIMS Kyoto Univ., 2003, pp. 1-14.

3. A. O. L. Atkin and J. Lehner, Hecke operators on $\Gamma_{0}(m)$, Math. Ann. 185 (1970), 134-160. MR.0268123 (42:3022)

4. H. Avelin, Deformation of $\Gamma_{0}(5)$-cusp forms, Math. Comp. 76 (2007), no. 257, 361-384 (electronic). MR2261026

5. A. Biró, Cycle integrals of Maass forms of weight 0 and fourier coefficients of Maass forms of weight 1/2, Acta Arithmetica 94 (2000), no. 2, 103-152. MR1779112 (2001g:11062)

6. A. Booker, A. Strömbergsson, and A. Venkatesh, Effective Computations with Maass Forms, In preparation.

7. Z.I. Borevich and I.R. Shafarevich, Zahlentheorie, Birkhäuser, 1966. MR.0195802 (33:4000)

8. R. W. Bruggeman, Modular forms of varying weight. I, Math. Z. 190 (1985), no. 4, 477-495. MR 808915 (87c:11051)

9. _ Modular forms of varying weight. II, Math. Z. 192 (1986), no. 2, 297-328. MR840831 $(87 \mathrm{k}: 11059)$

10. , Modular forms of varying weight. III, J. Reine Angew. Math. 371 (1986), 144-190. MR 859324 (88e:11040)

11. _ Families of automorphic forms, Birkhäuser, 1994. MR1306502 (95k:11060)

12. D. Bump, The Rankin-Selberg method: a survey, Number Theory, Trace Formulas and Discrete Groups (K.E. Aubert et al., ed.), Academic Press, 1989, pp. 49-109. MR993311 (90m:11079)

13. D. Bump and J. Hoffstein, On Shimura's correspondence, Duke Math. J. 55 (1987), no. 3, 661-691. MR904946 (89c:11072)

14. W. Duke, Hyperbolic distribution problems and half-integral weight Maass forms, Invent. Math. 92 (1988), 73-90. MR931205 (89d:11033)

15. A. Erdélyi, W. Magnus, F. Oberhettinger, and F. G. Tricomi, Higher Transcendental Functions. Vols. I, II, McGraw-Hill, 1953. MR0058756 (15:419i)

16. D. W. Farmer and S. Lemurell, Deformations of Maass forms, Math. Comp. 74 (2005), no. 252, 1967-1982 (electronic). MR 2164106

17. D.W. Farmer and S. Lemurell, Maass forms and their L-functions, arXiv:math.NT/0506102.

18. R. C. Gunning, Lectures on Modular Forms, Princeton University Press, 1962. MR0132828 (24:A2664)

19. G.H. Hardy, On the representation of a number as a sum of any number of squares and in particular five, Trans. Amer. Math. Soc. 21 (1920), 255-284. MR.1501144

20. D. A. Hejhal, Some Dirichlet series with coefficients related to periods of automorphic eigenforms. I,II, Proc. Japan Acad. Ser. A 58 (1982), 413-417; 59 (1983), 335-338.

21. The Selberg Trace Formula for PSL $(2, \mathbb{R})$, Vol.1, Lecture Notes in Mathematics, vol. 548, Springer-Verlag, 1976. MR0439755 (55:12641)

22. 1001, Springer-Verlag, 1983.

23. — A continuity method for spectral theory on Fuchsian groups, Modular forms (R.A. Rankin, ed.), Ellis-Horwood, Chichester, 1984, pp. 107-140. MR803365 (87g:11063)

24. _ Regular b-groups, degenerating Riemann surfaces, and spectral theory, Mem. Amer. Math. Soc. 88 (1990), no. 437, iv+138. MR.1052555(92h:11043) 
25. _ Eigenvalues of the Laplacian for Hecke triangle groups, Mem. Amer. Math. Soc. 97 (1992), no. 469, vi+165. MR1106989 (93f:11043)

26. - On eigenfunctions of the Laplacian for Hecke triangle groups, Emerging applications of number theory (D. Hejhal, J. Friedman, et al., eds.), IMA Vol. Math. Appl., vol. 109, Springer, New York, 1999, pp. 291-315. MR1691537(2000f:11063)

27. - On the Calculation of Maass Cusp Forms, Proceedings of the "International School on Mathematical Aspects of Quantum Chaos II", Lecture Notes in Physics, Springer, 2004, to appear.

28. H. Iwaniec, Topics in Classical Automorphic Forms, American Mathematical Society, 1997. MR.1474964 (98::11051)

29. S. Katok and P. Sarnak, Heegner points, cycles and Maass forms, Israel Journal of Mathematics 84 (1993), 193-227. MR1244668 (94h:11051)

30. K. Khuri-Makdisi, On the Fourier coefficients of nonholomorphic Hilbert modular forms of half-integral weight, Duke Math. J. 84 (1996), 399-452. MR1404335 (97m:11059)

31. M.I. Knopp, Modular Functions in Analytic Number Theory, Markham Publishing Company, 1970. MR0265287 (42:198)

32. W. Kohnen, Modular forms of half-integral weight on $\Gamma_{0}(4)$, Math. Ann. 248 (1980), no. 3, 249-266. MR575942 (81j:10030)

33. - Newforms of half-integral weight, J. Reine Angew. Math. 333 (1982), 32-72. MR660784 (84b:10038)

34. , Fourier coefficients of modular forms of half-integral weight, Math. Ann. 271 (1985), no. 2, 237-268. MR.783554 (86i:11018)

35. W. Kohnen and D. Zagier, Values of L-series of modular forms at the center of the critical strip, Invent. Math. 64 (1981), no. 2, 175-198. MR629468 (83b:10029)

36. H. Kojima, Shimura correspondence of Maass wave forms with half integral weight, Acta Arithmetica 69 (1995), no. 4, 367-385. MR1318756 (96a:11044)

37. - On the fourier coefficients of Maass wave forms of half integral weight over an imaginary quadratic field, J. Reine Angew. Math. 526 (2000), 155-179. MR1778303(2001h:11054)

38. - On the Fourier coefficients of Hilbert-Maass wave forms of half integral weight over arbitrary algebraic number fields, J. Number Theory 107 (2004), no. 1, 25-62. MR2059949 (2005c:11055)

39. S. Lang, Algebraic Number Theory, second ed., Graduate Texts in Mathematics, vol. 110, Springer-Verlag, New York, 1994. MR.1282723 (95f:11085)

40. H. Maass, Über eine neue Art von nichtanalytischen automorphen Funktionen und die Bestimmung Dirichletscher Reihen durch Funktionalgleichungen, Math. Ann. 121 (1949), 141183. MR0031519 (11:163c)

41. _ Die differentialgleichungen in der theorie der elliptischen modulfunktionen, Math. Ann. 125 (1952), 235-263 (1953). MR0065583 (16:449c)

42. _ Lectures on Modular Functions of One Complex Variable, second ed., Tata Institute of Fundamental Research Lectures on Mathematics and Physics, vol. 29, Tata Institute of Fundamental Research, Bombay, 1983. MR734485 (85g:11034)

43. W. Magnus and R. Oberhettinger, F. Soni, Formulas and Theorems for the Special Functions of Mathematical Physics, Third enlarged edition, Springer-Verlag, 1966.

44. T. Miyake, Modular Forms, Springer-Verlag, 1997.

45. L. J. Mordell, On the representations of a number as a sum of an odd number of squares, Trans. Cambr. Phil. Soc. 22 (1919), 361-372.

46. T. Mühlenbruch, Systems of Automorphic Forms and Period Functions, Ph.D. thesis, Universiteit Utrecht, 2003.

47. S. Niwa, Modular forms of half integral weight and the integral of certain theta-functions, Nagoya Math. J. 56 (1975), 147-161. MR0364106 (51:361)

48. Shinji Niwa, On Shimura's trace formula, Nagoya Math. J. 66 (1977), 183-202. MR0562506 (58:27781)

49. S. J. Patterson, The Laplacian operator on a Riemann surface, Compositio Math. 31 (1975), no. 1, 83-107. MR0384702 (52:5575)

50. ㄴ. The Laplacian operator on a Riemann surface. II, Compositio Math. 32 (1976), no. 1, 71-112. MR0419364 (54:7385)

51. — The Laplacian operator on a Riemann surface. III, Compositio Math. 33 (1976), no. 3, 227-259. MR0491511(58:10750) 
52. H. Petersson, Theorie der automorphen formen beliebiger reeller dimension und ihre darstellung durch eine neue art Poincaréscher reihen, Math. Ann. 103 (1930), 369-436. MR.1512629

53. _ Zur analytischen Theorie der Grenzkreisgruppen. I. Grenzkreisgruppen und Riemannsche Flächen; Theorie der Faktoren- und Multiplikatorsysteme komplexer Dimension, Math. Ann. 115 (1937), 23-67.

54. — Automorphe Formen als metrische Invarianten. II. Multiplikative Differentiale als Grenzwerte metrischer Invarianten von stetig veränderlicher reeller Dimension, Math. Nachr. 1 (1948), 218-257. MR0028964(10:525g)

55. R. A. Rankin, Modular Forms and Functions, Cambridge University Press, 1976.

56. W. Roelcke, Das Eigenwertproblem der automorphen Formen in der hyperbolischen Ebene. I, II, Math. Ann. 167 (1966), 292-337; ibid. 168 (1966), 261-324. MR0243062 (39:4386)

57. P. Sarnak, Additive number theory and Maass forms, Number theory (New York, 1982), Lecture Notes in Math., vol. 1052, Springer, Berlin, 1984, pp. 286-309. MR750670 (86f:11042)

58. A. Selberg, Harmonic analysis and discontinuous groups in weakly symmetric Riemannian spaces with applications to Dirichlet series, J. Indian Math. Soc. (N.S.) 20 (1956), 47-87. MR.0088511 (19:531g)

59. G. Shimura, Introduction to the Arithmetic Theory of Automorphic Forms, Princeton Univ. Press, 1971.

60. - On modular forms of half integral weight, Ann. of Math. 97 (1973), 440-481. $\operatorname{MR} 0332663(48: 10989)$

61. On the Fourier coefficients of Hilbert modular forms of half-integral weight, Duke Math. J. 71 (1993), 501-557. MR 1233447 (94e:11046)

62. T. Shintani, On construction of holomorphic cusp forms of half integral weight, Nagoya Math. J. 58 (1975), 83-126. MR0389772 (52:10603)

63. H. M. Stark, Fourier coefficients of Maass waveforms, Modular forms (Durham, 1983), Ellis Horwood Ser. Math. Appl.: Statist. Oper. Res., Horwood, Chichester, 1984, pp. 263-269. MR $803370(87 \mathrm{~h}: 11128)$

64. F. Strömberg, Computational aspects of maass waveforms, Ph.D. thesis, Uppsala University, 2004.

65. - Maass waveforms on $\left(\Gamma_{0}(N), \chi\right)$, computational aspects, Proceedings of the "International School on Mathematical Aspects of Quantum Chaos II", 2005, to appear.

66. - Hecke Operators for Maass Waveforms on psl $(2, \mathbb{Z})$ with Integer Weight and Eta Multiplier, International Mathematics Research Notices (2007), article ID rnm062, 25 pages, doi:10.1093/imrn/rnm062.

67. A. Strömbergsson, The Selberg Trace Formula for $S L(2, \mathbb{R})$ and arbitrary real weight, unpublished manuscript.

68. - Studies in the analytical and spectral theory of automorphic forms, Ph.D. thesis, Uppsala University, Dept. of Math., 2001.

69. H. Then, Maass cusp forms for large eigenvalues, Math. Comp. 74 (2005), no. 249, 363-381. MR 2085897 (2005h:11106)

70. J. H. van Lint, Hecke operators and Euler products, Drukkerij "Luctor et Emergo", Leiden, 1957. MR 0090616 (19:839f)

71. sch. Proc. Ser. A. 61 = Indag. Math. 20 (1958), 522-527. MR0103287 (21:2065)

72. J. Von Neumann and E. Wigner, Über das Verhalten von Eigenwerten bei adiabatischen Processen, Phys. Zeit. 30 (1929), 467-470.

73. J.-L. Waldspurger, Correspondance de Shimura, J. Math. Pures Appl. (9) 59 (1980), no. 1, 1-132. MR:577010 (83f:10029)

74. Sur les coefficients de Fourier des formes modulaires de poids demi-entier, J. Math. Pures Appl. (9) 60 (1981), no. 4, 375-484. MR646366 (83h:10061)

75. A. M. Winkler, Cusp forms and Hecke groups, J. Reine Angew. Math. 386 (1988), 187-204. MR 936998 (90g:11067)

76. K. Wohlfahrt, Über Operatoren Heckescher Art bei Modulformen reeller Dimension, Math. Nachr. 16 (1957), 233-256. MR0106888 (21:5618) 
Institut für Theoretische Physik, TU Clausthal, Abteilung Statistische Physik und Nichtlineare Dynamik, Arnold-Sommerfeld-Strasse-6, 38678 Clausthal-Zellerfeld, GERMAnY

Current address: Fachbereich Mathematik, TU Darmstadt, Schlossgartenstrasse 7, 64289 Darmstadt, Germany

E-mail address: stroemberg@mathematik.tu-darmstadt.de 